\title{
Kinetochore- and chromosome-driven transition of microtubules into bundles promotes spindle assembly
}

\author{
Jurica Matković ${ }^{\text {, Subhadip Ghosh }}{ }^{2}$, Mateja Ćosić ${ }^{1}$, Nenad Pavin ${ }^{2}$, Iva M. Tolić ${ }^{*}$ \\ ${ }^{1}$ Division of Molecular Biology, Ruđer Bošković Institute, Bijenička cesta 54, 10000 Zagreb, \\ Croatia \\ ${ }^{2}$ Department of Physics, Faculty of Science, University of Zagreb, Bijenička cesta 32, 10000 \\ Zagreb, Croatia \\ *Correspondence to: tolic@irb.hr
}

\section{ABSTRACT}

Mitotic spindle assembly is crucial for chromosome segregation and relies on bundles of microtubules that extend from the poles and overlap in the middle. However, how these structures form remains poorly understood. Here we show that overlap bundles arise through a network-to-bundles transition driven by kinetochores and chromosomes. STED superresolution microscopy revealed that PRC1-crosslinked microtubules initially form loose arrays, which become rearranged into bundles. Kinetochores promote microtubule bundling by lateral binding via CENP-E/kinesin-7 in an Aurora B-regulated manner. Bundle separation is driven by steric interactions of the bundle-associated chromosomes at the spindle midplane since spindles with uncondensed or uncongressed chromosomes are narrower. In agreement with experiments, theoretical modeling suggests that bundles arise through competing attractive and repulsive mechanisms. Finally, perturbation of overlap bundles led to inefficient correction of erroneous kinetochore-microtubule attachments. Thus, kinetochores and chromosomes drive coarsening of a uniform microtubule array into overlap bundles, which promote not only spindle formation but also chromosome segregation fidelity. 


\section{INTRODUCTION}

36 Segregation of chromosomes during mitosis relies on the mitotic spindle, a supramolecular micro-machine made of microtubules and numerous microtubule-associated proteins, which is built de novo in each cell cycle ${ }^{1}$. While some spindle microtubules exist as individual filaments, such as astral microtubules, many are organized in bundles, such as kinetochore fibers and overlap bundles ${ }^{2,3}$. Kinetochore fibers are bundles of parallel microtubules that attach in an end-on manner to the kinetochore on the centromere of each chromosome, and link the kinetochore with the spindle pole ${ }^{4,5}$. Overlap or interpolar bundles consist of antiparallel microtubules that extend from the opposite spindle halves ${ }^{6}$. Spindle assembly has been extensively studied with focus on kinetochore dynamics and the formation of kinetochore fibers $^{7-12}$, whereas the mechanism of overlap bundle formation remains poorly understood.

Overlap bundles are essential for spindle assembly, as inhibition of the Eg5/kinesin-5 motor protein, which slides apart the antiparallel microtubules within the bundle, leads to monopolar spindles ${ }^{13-16}$. Such spindles without overlap bundles cannot segregate the chromosomes even though they usually contain kinetochore fibers, highlighting the indispensable role of overlap bundles in spindle functioning. In a mature spindle in metaphase, overlap bundles link sister kinetochore fibers like a bridge and because of this interaction are known as bridging fibers ${ }^{17}$. Their functions include balancing the tension on kinetochores ${ }^{17-21}$, promoting chromosome alignment at the spindle midplane 22,23 and driving spindle elongation and chromosome segregation in anaphase ${ }^{24-28}$. Microtubules within bridging fibers are linked by the protein regulator of cytokinesis 1 (PRC1) ${ }^{17,21,29}$, a crosslinker that has a 10-fold preference for antiparallel versus parallel microtubule overlaps in vitro ${ }^{30-33}$. Such crosslinkers together with motor proteins may drive the formation of initial overlaps between two antiparallel microtubules, as shown by computer simulations ${ }^{34-37}$.

Mitotic spindles of human cells are made of more than 6000 microtubules $^{38}$. In general, microtubules present in large numbers can exist in different phases, from loose networks to tight bundles, where the networks can be isotropic if the filaments are unoriented, nematic if the filaments are parallel and point either way, or polar if they point in a common direction ${ }^{39}$. Different types of microtubule networks have been studied in vitro ${ }^{40-42}$, together with theoretical studies that explored under what conditions certain types of polymer networks appear or undergo a transition to bundles ${ }^{43-47}$. Yet, whether similar transitions occur in cells and what biological function they may have are open questions. 
In this work we develop an assay for bundle formation, together with a theoretical model, and show that microtubules crosslinked by PRC1 undergo a network-to-bundles transition during early stages of spindle assembly. We find that this transition is stimulated by kinetochores, which promote microtubule bundling by binding to them laterally via CENP-E in a manner regulated by Aurora B. We further show that the separation of the bundles, which leads to spindle widening and the characteristic spindle shape, is driven by steric interactions of the chromosomes bound to the bundles as they congress to the spindle midplane, based on our findings that spindles with uncondensed or incompletely congressed chromosomes are narrower. Moreover, we identify a function of overlap bundles in the correction of erroneous kinetochore-microtubule attachments. Our work thus reveals that kinetochores and chromosomes together with crosslinkers drive coarsening of an initially uniform microtubule array into neatly organized overlap bundles, which not only support spindle assembly but also promote error-free mitosis.

\section{RESULTS}

The network of PRC1-crosslinked microtubules undergoes a transition to bundles

The overarching question of how the cell generates microtubule bundles that form the spindle shape contains two parts: how individual bundles are formed and what determines their separation. To study individual bundle formation, we first explored microtubule organization during early stages of mitosis by using stimulated emission depletion (STED) microscopy ${ }^{48}$ to obtain super-resolution images of microtubules, together with the microtubule crosslinker PRC1 and chromosomes (Fig. 1a). The spatial pattern of microtubules and PRC1 changed profoundly during spindle formation and maturation (Fig. 1a). In early prometaphase characterized by the "prometaphase rosette" where the chromosomes are arranged like flower petals along the edge of the nascent spindle ${ }^{49,50}$, the majority of spindle body microtubules appeared as a diffuse array with a few bundles present mainly at the edges ("Rosette" in Fig. 1a and Extended Data Fig. 1a). During late prometaphase, when chromosomes increasingly congress to the equatorial plane, and afterwards in metaphase, additional microtubule bundles appeared and their spacing became more regular, which was accompanied by the appearance of PRC1 stripes along the bundles ("Congression" and "Metaphase" in Fig. 1a and Extended Data Fig. 1a). 
By looking at cross-sections of vertically oriented spindles, we found that microtubules of the spindle body are initially uniformly distributed over a ring-shaped region, and astral microtubules extend radially outwards (Fig. 1a end-on view). The spindle body microtubules became rearranged into discrete bundles that fill the spindle cross-section at an even spacing (end-on view in Fig. 1a and Extended Data Fig. 1a). Similarly, PRC1 distribution changed from a nearly homogeneous scattering over the ring-shaped region, which we call PRC1 network, to spot-like structures that colocalized with the microtubule bundles (end-on view in Fig. 1a and Extended Data Fig. 1a).

To study how a loose microtubule array transforms into bundles, we developed a livecell assay termed "bundling assay" based on PRC1 as a marker of microtubule overlaps, where we imaged cross-sections of vertically oriented spindles in HeLa cells expressing PRC1-GFP starting at the rosette stage for 10 minutes (Fig. 1b). The rosette was identified by a chromosome circle surrounding the spindle, which was not yet fully closed (Extended Data Fig. $1 \mathrm{~b}$ top and Fig. 1a bottom left). Time-lapse images revealed that the spatial distribution of PRC1 in the spindle cross-section gradually changes from a homogeneous dispersion over a ring-shaped area (PRC1 network) to discrete clusters (Fig. 1b and Supplementary Video 1), in agreement with STED images of fixed cells. Segmentation analysis (Methods; Extended Data Fig. 1c,d) showed that the number of PRC1-labeled segments and their mean PRC1-GFP intensity increased over time, whereas the segment area decreased (Fig. 1c,d and Extended Data Fig. 1e).

Intensity profiles of $\mathrm{PRC} 1$ along the ring-shaped area indicate that in comparison with the nearly constant PRC1 intensity in the rosette, the intensity at a later stage exhibited higher peaks and deeper valleys ( $\mathrm{t}=0$ vs. 7.2 minutes, Fig. 1e,f). The mean intensity did not change substantially, but the standard deviation increased 2.6-fold ( $\mathrm{n}=10$, Fig. $1 \mathrm{~g}$ and Extended Data Fig. 1f). This suggests that the nearly uniform PRC1-labeled microtubule network undergoes a transition to bundles that accumulate PRC1, leaving the space between the bundles almost free from PRC1.

We next asked how the initial PRC1 network is formed. A large fraction of spindle microtubules is nucleated by the augmin complex at the wall of other microtubules ${ }^{51,52}$. When

130 we used the bundling assay on cells depleted of the augmin subunit Haus6, we found that, in

131 contrast to untreated cells, a PRC1 network was absent at the rosette stage (Extended Data Fig. 132 1g-i and Supplementary Video 2) and the dynamics of bundle formation was slower (Extended 133 Data Fig. 1j-1). These results suggest that the initial array of microtubules crosslinked by PRC1 134 is largely nucleated by the augmin complex. 
To verify that the redistribution of PRC1 indeed reflects changes of the microtubule

136 network, which was observed in STED images, we first used nocodazole to depolymerize

137 microtubules at the rosette stage. This treatment caused the disappearance of the PRC1 network

138 (Extended Data Fig. 2a), indicating that PRC1 forming this structure is bound to microtubules.

139 Second, we noticed that PRC1 colocalized with microtubules in the central part of the spindle

140 but was excluded from astral microtubules throughout prometaphase (Fig. 1a and Extended

141 Data Fig. 2b), suggesting that PRC1 selectively localizes to antiparallel microtubule overlaps

142 already in early mitosis. Third, we imaged spindles stained with SiR-tubulin and found that,

143 similarly to PRC1, tubulin signal undergoes a change from a nearly uniform to a spotted

144 distribution (Extended Data Fig. 2c,d). Yet, PRC1 is a better marker of antiparallel overlaps

145 not only because of its specificity but also because of the lower background signal (Fig. 1b vs.

146 Extended Data Fig. 2c). Moreover, an increase in the number of bundles during prometaphase

147 was observed in horizontally oriented HeLa spindles labeled with PRC1-GFP, as well as in

148 spindles of human non-transformed retinal pigment epithelial hTERT-RPE1 cells stained with

149 SiR-tubulin (Extended Data Fig. 2e), suggesting that the process of bundle formation is

150 independent of the cell line and labeling. Taken together, these results confirm that the PRC1

151 redistribution reflects the redistribution of overlap microtubules from a nearly uniform network

152 in early prometaphase to the regularly spaced overlap bundles in metaphase. 
bioRxiv preprint doi: https://doi.org/10.1101/2022.02.25.481924: this version posted February 25, 2022. The copyright holder for this preprint (which was not certified by peer review) is the author/funder, who has granted bioRxiv a license to display the preprint in perpetuity. It is made available under aCC-BY-NC-ND 4.0 International license.

Figure 1
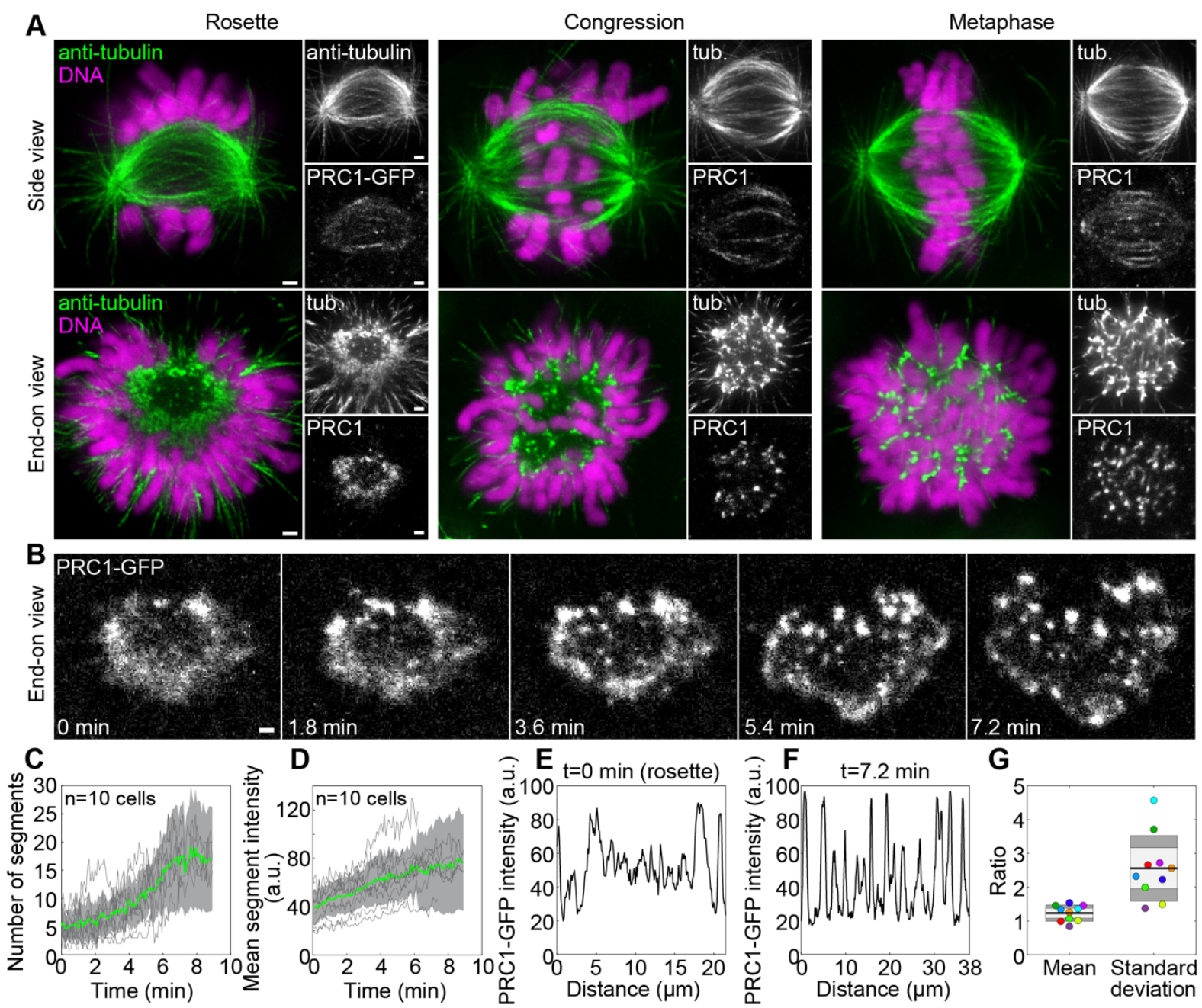

Fig. 1: PRC1-crosslinked microtubules undergo a transition from a loose array to bundles during spindle assembly.

158 a, Spindles immunostained for $\alpha$-tubulin and imaged at STED resolution in a HeLa-Kyoto BAC cell line stably expressing PRC1-GFP with DNA stained by DAPI (both imaged at a confocal resolution), in early prometaphase

160 ("Rosette"), late prometaphase ("Congression"), and metaphase. Spindles lying parallel (first row) or perpendicular (second row) to the imaging plane are shown. $\mathbf{b}$, Time lapse images (single plane) of a cross-section of a vertically oriented prometaphase spindle in a HeLa-Kyoto BAC cell stably expressing PRC1-GFP, starting with the prometaphase rosette. $\mathbf{c}$, Number of PRC1 segments in the midplane over time for cells as in panel $\mathbf{b}$, obtained using Squash segmentation. Gray lines represent individual cells, green line the mean and gray areas the standard deviation, $\mathrm{n}=10$ cells in 10 independent experiments. $\mathbf{d}$, Mean intensity of $\mathrm{PRC} 1$ segments from the same cells as in c. e,f, Intensity line plots of PRC1-GFP in the cell from panel $\mathbf{b}$ at $t=0$ (e) and $t=7.2 \mathrm{~min}$ (f) along the periphery of the spindle cross-section (lines shown in Extended Data Fig. 1f). g, Ratio of mean PRC1-GFP intensity in panels $\mathbf{f}$ and $\mathbf{e}$ ("Mean") and ratio of the standard deviation in panels $\mathbf{f}$ and $\mathbf{e}$ ("Standard deviation"). The black line shows the mean; the light and dark grey areas mark 95\% confidence interval on the mean and 
standard deviation, respectively; $\mathrm{n}=10$ cells in 10 independent experiments. Dots of the same color represent the same cells. All scale bars, $1 \mu \mathrm{m}$.

Kinetochores promote overlap bundle formation by CENP-E-mediated attachments in an Aurora B-dependent manner

In metaphase, overlap microtubule bundles are found close to a pair of sister kinetochores ${ }^{53,54}$. This finding inspired us to ask whether kinetochores play a role in bundle formation during early prometaphase (Fig. 2a), a stage when kinetochores are laterally attached to spindle microtubules ${ }^{49,50,55,56}$. We found that, in a rosette cross-section of unperturbed spindles, most of the PRC1-labeled bundles had an adjacent kinetochore at its outer side (Fig. 2b). The bundles with an associated kinetochore had a higher PRC1 signal intensity than those without a kinetochore (Fig. 2c), consistent with the hypothesis that kinetochores promote microtubule bundling.

To explore the role of lateral attachments of kinetochores in bundle formation, we inhibited Aurora B, which is in prometaphase localized mainly at centromeres (Extended Data Fig. 3a) and required for the lateral attachment ${ }^{50,57-59}$. We first explored the effect of Aurora $B$ inhibition by barasertib (AZD1152-HQPA) ${ }^{60,61}$ on mature bundles in metaphase. Strikingly, we found a 4.2-fold decrease in the signal intensity of individual PRC1-labeled bundles in the cross-section of metaphase spindles ( $\mathrm{n}=452$ bundles from 14 cells, Fig. 2d,e; Extended Data Fig. $3 b$ and side view in Extended Data Fig. 3c), suggesting that antiparallel bundles contained fewer microtubules. To confirm this, we measured the tubulin signal intensity in the central part of individual bridging fibers in the region between sister kinetochores in metaphase and found a 7.6-fold decrease upon Aurora B inhibition (Fig. 2f,g and Extended Data Fig. 3d). Thus, Aurora B activity regulates the number of microtubules within the bridging fibers in metaphase.

We addressed the role of Aurora B from another angle by exploring localization relationships between Aurora B and PRC1 in metaphase. We found that the signal intensity of PRC1 within the bridging fiber is correlated with the signal of Aurora B in the neighboring centromere (Extended Data Fig. 3e). Moreover, when we displaced Aurora B from the inner centromere to a position closer to the kinetochore by transfecting cells with CENP-B-INCENP$\mathrm{GFP}^{62-64}$, we observed a remarkable change in the PRC1 signal distribution. Instead of a single broad PRC1 peak in the central part of the spindle that is evident in untreated cells, the cells with displaced Aurora B showed two PRC1 peaks about $1 \mu \mathrm{m}$ away from the spindle equator 
bioRxiv preprint doi: https://doi.org/10.1101/2022.02.25.481924; this version posted February 25, 2022. The copyright holder for this preprint (which was not certified by peer review) is the author/funder, who has granted bioRxiv a license to display the preprint in perpetuity. It is made available under aCC-BY-NC-ND 4.0 International license.

204

205

206

207

208

209

210

211

212

on either side (Fig. 2h,i and Extended Data Fig. 3f). These results suggest that Aurora B regulates the localization of PRC1-labeled bundles within the spindle.

To test the effect of Aurora $\mathrm{B}$ on the dynamics of bundle formation during early prometaphase, we combined the bundling assay with acute inhibition of Aurora B by barasertib added at the rosette stage (Fig. $2 \mathrm{j}$ and Supplementary Video 3). Aurora B inhibition led to a slower increase in the number and intensity of PRC1-labeled bundles over time in comparison with untreated cells (Extended Data Fig. 3g,h), which confirmed that Aurora B regulates the dynamics of bundle formation.

Figure 2

A
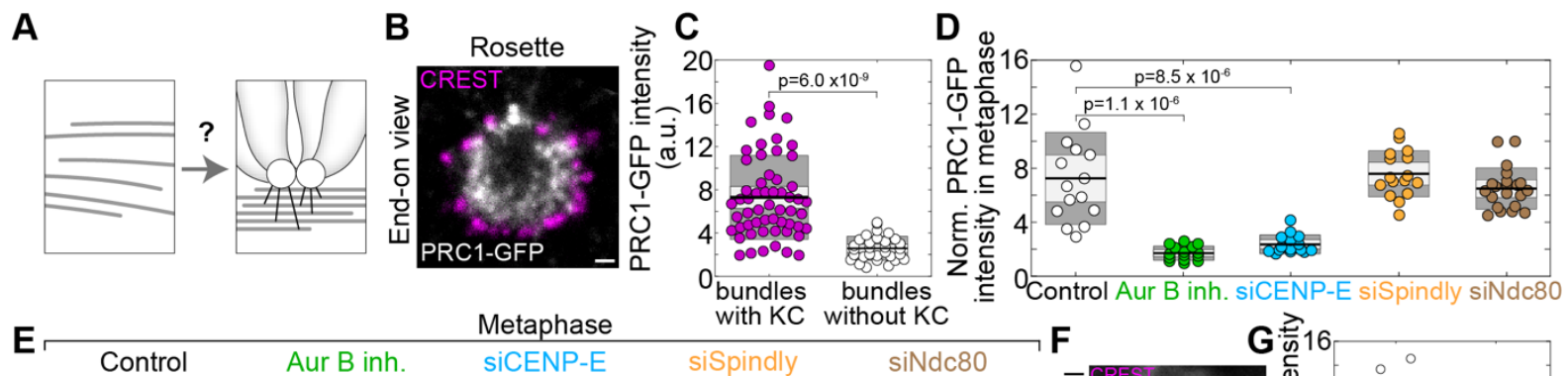

Metaphase $\begin{array}{ll}\text { with KC } \text { without KC } \\ \text { sindly } & \text { siNdc80 }\end{array}$
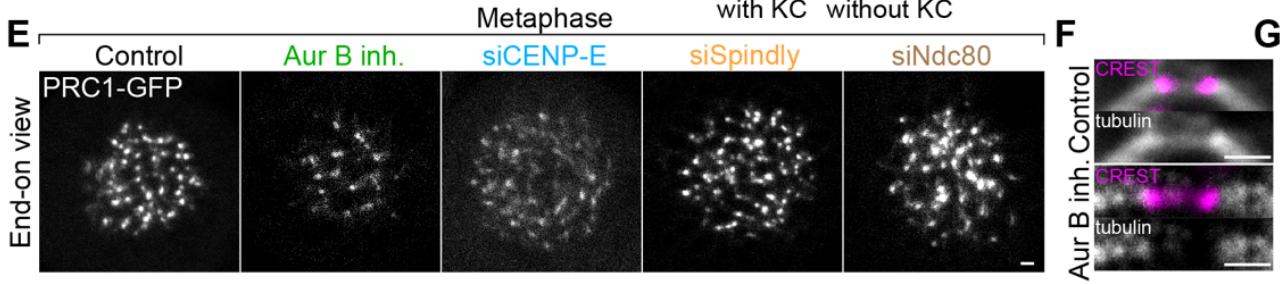

$J_{\sqrt{ }}$ Aurora B inhibitor barasertib added
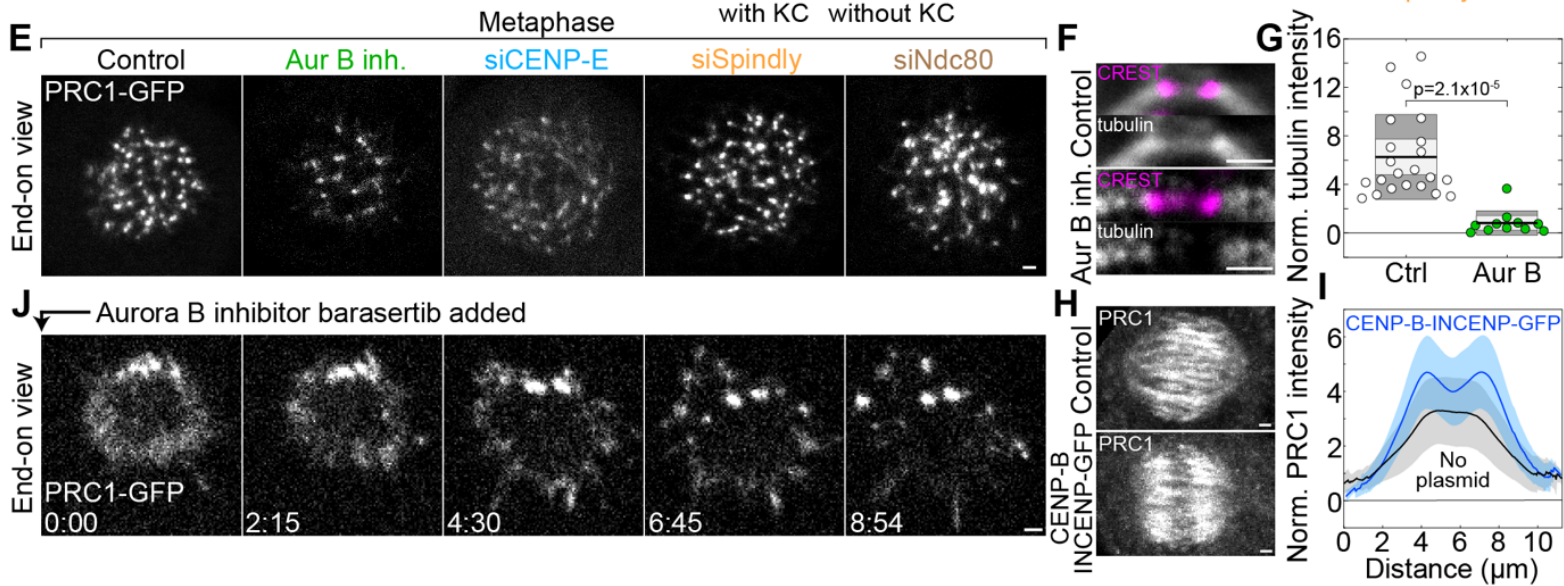

Fig. 2: Kinetochores promote microtubule bundle formation in an Aurora B-dependent manner.

216 a, Schematic view of kinetochore-driven overlap bundle formation. b, Midplane of a vertically oriented prometaphase rosette immunostained for CREST (magenta) in a HeLa-Kyoto BAC cell line stably expressing PRC1-GFP (white). c, PRC1-GFP intensity of bundles with and without adjacent kinetochore, $\mathrm{n}=57$ bundles with kinetochores attached and 30 without, in 10 cells from 3 independent immunostainings. d, PRC1-GFP intensity of bundles in vertically oriented metaphase spindles, normalized to the cytoplasmic PRC1-GFP intensity. Circles represent the mean bundle intensity per spindle. One-way ANOVA test showed significant difference between group means, p-values are given for the significant differences from control. Numbers of bundles/cells from left to right: $827 / 15,452 / 14,730 / 15,880 / 16,774 / 21 ; 3$ independent experiments per condition. e, Images of vertically oriented metaphase spindles in a HeLa-Kyoto BAC cell line stably expressing PRC1-GFP (white), treated as indicated. $\mathbf{f}$, Image of a region around a kinetochore pair immunostained for CREST (magenta) and tubulin (white) in an untransfected HeLa cell, control (top) and after Aurora B inhibition (bottom). Merged channels and only 
tubulin are shown. $\mathbf{g}$, Mean tubulin intensity in the middle of the bridging fiber in control and Aurora B-inhibited cells, normalized to the cytoplasmatic anti-tubulin intensity; $\mathrm{n}=22$ bundles from 14 cells for control and 11 bundles from 9 cells for Aurora B inhibition, from 3 independent immunostainings. h, Maximum-intensity projections of 41 planes of metaphase spindles immunostained for PRC1 (white) in an untransfected HeLa cell line (control, top image), and cells transfected with CENP-B-INCENP-GFP (bottom image). i, Line intensity profiles of the antiPRC1 signal intensity, normalized to the cytoplasmatic anti-PRC1 signal intensity, along the spindle main axis, for control ( $\mathrm{n}=33$ ) and treated cells as in $\mathbf{h}(\mathrm{n}=30)$, mean (central line) and standard deviation (colored surfaces), 3 independent experiments per condition. $\mathbf{j}$, Time lapse images (single plane) of a cross-section of a vertically oriented prometaphase spindle in a HeLa-Kyoto BAC cell stably expressing PRC1-GFP, after addition of the Aurora B inhibitor Barasertib at the prometaphase rosette stage. In $\mathbf{c}, \mathbf{d}$ and $\mathbf{g}$ the black line shows the mean; the light and dark grey areas mark $95 \%$ confidence interval on the mean and standard deviation, respectively. All scale bars, $1 \mu \mathrm{m}$.

The mechanism by which Aurora B at the kinetochore promotes microtubule bundling likely relies on Aurora B substrates involved in microtubule binding. We hypothesize that if microtubule-binding proteins that are localized at the kinetochore in multiple copies attach to several microtubules positioned close to the kinetochore, this could promote microtubule bundling (Fig. 3a). Candidates for this activity are two motor proteins, CENP-E (kinesin-7) and cytoplasmic dynein, and the outer kinetochore protein $\mathrm{Ndc} 80$, given that they bind to microtubules, localize at the kinetochore in prometaphase (CENP-E and dynein) or throughout mitosis (Ndc80), and are regulated by Aurora $\mathrm{B}^{50,65-67}$.

We first analyzed the signal intensity of PRC1-labeled bundles in metaphase after depletion of these candidates. CENP-E depletion by siRNA resulted in a 3.1-fold lower intensity of PRC1 in individual bundles in metaphase ( $\mathrm{n}=730$ bundles from 14 cells, Fig. 2d,e and Extended Data Fig. 3b,c; see Extended Data Fig. 4 for depletion efficiency) and a 2.8-fold decrease in the tubulin signal intensity in the central part of individual bridging fibers (Fig. 3b,c and Extended Data Fig. 3d). These results suggest that CENP-E is required for proper bundle formation and motivated us to explore its localization and dynamics during early prometaphase. In the rosette, we found that CENP-E localizes at the side of the kinetochore facing the PRC1-labeled bundles (Fig. 3d,e), which suggests that it links the kinetochore with the antiparallel bundles. The bundling assay revealed that acute inhibition of CENP-E by GSK$923295^{68}$ at the rosette stage led to a slower increase in the number and intensity of PRC1labeled bundles over time than in untreated cells (Fig. 3f, Extended Data Fig. 3i,j and Supplementary Video 4), supporting a role of CENP-E in bundle formation. Finally, the hypothesis that CENP-E promotes bundling predicts that overexpression of CENP-E should lead to increased bundling, which we indeed observed as a higher intensity of PRC1-labeled 
bioRxiv preprint doi: https://doi.org/10.1101/2022.02.25.481924; this version posted February 25, 2022. The copyright holder for this preprint (which was not certified by peer review) is the author/funder, who has granted bioRxiv a license to display the preprint in perpetuity. It is made available under aCC-BY-NC-ND 4.0 International license.

bundles in metaphase upon CENP-E overexpression (Fig. 3g,h and Extended Data Fig. 3k).

264 Taken together, these results indicate that kinetochores promote bundle formation via CENP-

265 E-mediated microtubule binding.

To test whether dynein at the kinetochore is required for overlap bundle formation, we depleted Spindly, a kinetochore-specific adaptor for dynein that recruits dynein to the kinetochore ${ }^{69}$. Spindly depletion did not change the signal intensity of PRC1-labeled bundles in metaphase (Fig. 2d,e and Extended Data Fig. 3b; see Extended Data Fig. 4 for depletion efficiency), suggesting that dynein is not crucial for overlap bundle formation. Similarly, Ndc80 depletion did not alter bundle intensity (Fig. 2d,e and Extended Data Fig. 2b; see Extended Data Fig. 4 for depletion efficiency), arguing against a major role of $\mathrm{Ndc} 80$ in overlap bundle formation. Moreover, as Ndc80 is required for the establishment of end-on attachments of kinetochores to microtubules and thus the formation of kinetochore fibers ${ }^{57,66,70,71}$, these data imply that overlap microtubule bundles can form independently of kinetochore fibers.

A

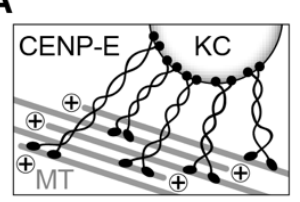

B

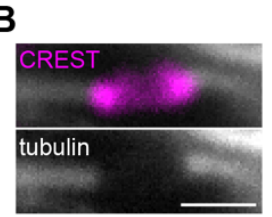

F CENP-E inhibitor GSK923295 added

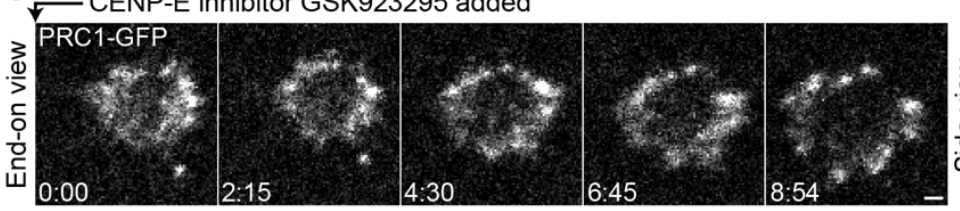

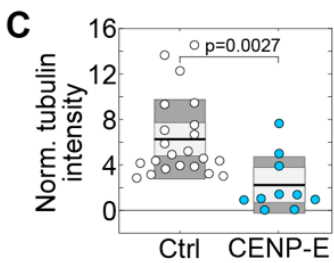

IRNA
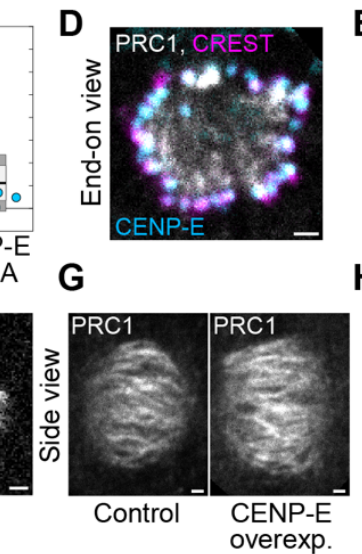

Fig. 3: CENP-E promotes overlap bundle formation.

a, Schematic view of how CENP-E may promote microtubule bundling. b, Image of a region close to kinetochores immunostained for CREST (magenta) and tubulin (white) in a CENP-E depleted HeLa cell. c, Mean tubulin intensity in the middle of the bridging fiber in control and CENP-E depleted cells, normalized to the cytoplasmatic tubulin intensity, $\mathrm{n}=22$ bundles from 14 cells for control and 10 bundles from 7 cells for CENP-E depletion, from 3 independent immunostainings. d, Cross-section of a vertically oriented prometaphase rosette immunostained for CREST (magenta) and CENP-E (cyan) in a HeLa-Kyoto BAC cell line stably expressing PRC1-GFP (white). e, Line intensity plots of PRC1-GFP, CREST and CENP-E from the spindle shown in d, normalized to the corresponding maximum value. The line was drawn across the middle of the spindle cross-section, with a line thickness of $1 / 3$ of the spindle cross-section. $\mathbf{f}$, Time lapse images (single plane) of a cross-section of a vertically 
oriented prometaphase spindle in a HeLa-Kyoto BAC cell stably expressing PRC1-GFP (white), after addition of the CENP-E inhibitor GSK923295 at the prometaphase rosette stage. g, Images of horizontally oriented metaphase spindles immunostained for PRC1 (white) in an untransfected HeLa cell line (control, left) and after CENP-E overexpression (right). $\mathbf{h}$, Total PRC1 intensity on the spindle in control $(\mathrm{n}=41)$ and CENP-E overexpression $(\mathrm{n}=30)$, from 3 immunostainings. In $\mathbf{c}$ and $\mathbf{h}$, the black line shows the mean; the light and dark grey areas mark 95\% confidence interval on the mean and standard deviation, respectively. All scale bars, $1 \mu \mathrm{m}$.

\section{Steric interactions of the chromosomes separate the bundles and expand spindle width}

As the bundles form, they undergo characteristic movements. We first focused on the bundles that connect the centrosomes in a straight line, which can be identified by the highest PRC1 signal and a lack of an attached chromosome at the rosette stage (see Fig 1a). These bundles are typically found at the edge of the rosette and become centrally positioned over a period of about 5 minutes (Fig. 4a, images to the left). We traced the movement of all bundles and found that, in a coordinate system centered at the brightest bundles, the bundles forming adjacent to them move outwards and encircle them, whereas the bundles forming on the opposite side of the cross-section move away from the brightest bundles (Fig. 4a, traces and Supplementary Video 5). As this movement is accompanied by the arrival of more and more chromosomes to the spindle midplane, we hypothesized that chromosomes promote bundle separation and thus spindle width expansion by steric effects (crowding) upon congression to the midplane. Indeed, we found that as new chromosomes arrived at the midplane, the chromosomes that were already there moved away from the site of the new arrival (Fig. $4 \mathrm{~b}$ left). This led to the overall outward movement of the PRC1-labeled bundles and spindle widening (Fig. 4b right and Supplementary

313 Video 6). When looking at the spindles in a side view, we found that the spindle width increases

314 as increasingly more chromosomes arrive to the metaphase plate (Fig. 4c,d and Supplementary

315 Video 7). These results support the hypothesis that chromosome crowding increases bundle separation.

This hypothesis predicts that removing the chromosomes from the spindle and/or 318 decondensing the chromatin should result in narrower spindles. To test this idea, we induced 319 mitosis with unreplicated genome (MUG) by treating the cells with hydroxyurea to inhibit 320 DNA replication and caffeine to override the DNA damage checkpoint ${ }^{10,72}$, because in such cells the bulk of the uncondensed chromatin remains away from the spindle, while the kinetochores are attached to the spindle microtubules ${ }^{10}$. We found that the MUG spindles were significantly narrower with more tightly packed bundles than spindles in untreated cells (Fig. 4e,f). 
bioRxiv preprint doi: https://doi.org/10.1101/2022.02.25.481924; this version posted February 25, 2022. The copyright holder for this preprint (which was not certified by peer review) is the author/funder, who has granted bioRxiv a license to display the preprint in perpetuity. It is made available under aCC-BY-NC-ND 4.0 International license.

Another prediction of the chromosome crowding hypothesis is that if the chromosomes

326 are not aligned at the equator but distributed over the spindle, the spindles should be narrower,

327 which we tested by several protein depletions. Ndc80 depletion, which results in a large

328 fraction of chromosomes distributed along the spindle due to the absence of kinetochore

329 fibers $^{70,73,74}$, led to narrower spindles than in untreated cells (Fig. 4e,f). To test chromosome

330 misalignment without abolishing kinetochore fibers, we used depletion of Kif18A (kinesin-8),

331 a motor protein that regulates chromosome oscillations around the spindle midplane, whose

332 depletion leads to extensive oscillations and hence chromosome scattering along the

333 spindle $\mathrm{e}^{75,76}$. In this case we also found narrower spindles ${ }^{77}$ (Fig. 4f). To further test our

334 hypothesis, we divided the cells depleted of CENP-E into two groups: those with high

335 chromosome misalignment, where more than $50 \%$ of the total chromosome mass was found

336 away from the spindle midplane, and low misalignment where 1-5 chromosomes were

337 misaligned. CENP-E depleted spindles with high misalignment were narrower than those with

338 low misalignment (Fig. 4e,f). Taken together, these results support the hypothesis that

339 chromosome accumulation on the metaphase plate promotes the separation of the associated

340 microtubule bundles and spindle width expansion.

Figure 4

A

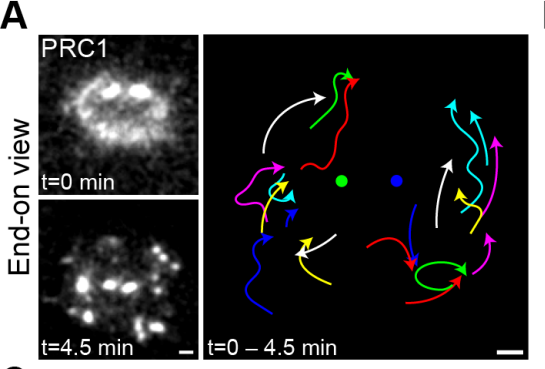

B
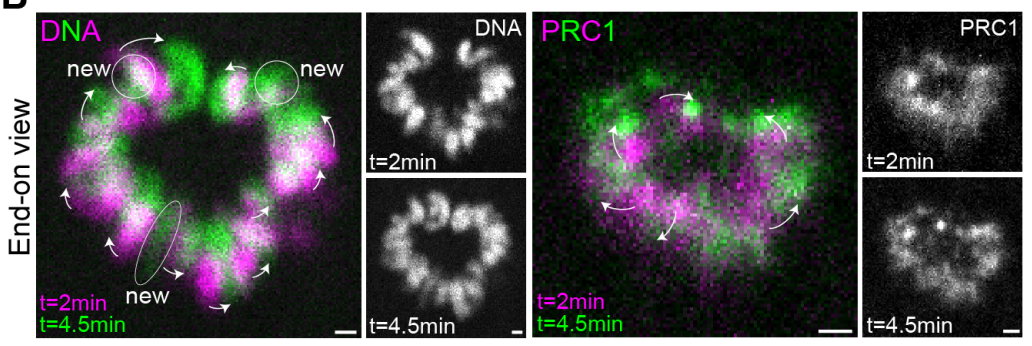

D
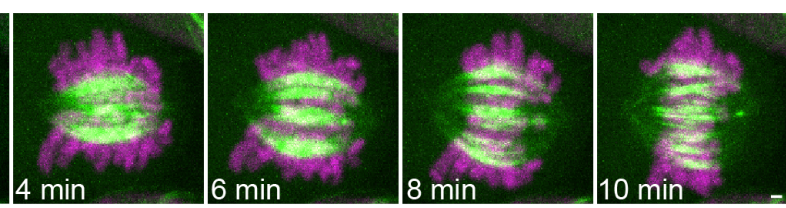

E $\begin{gathered}\text { Control } \\ \text { (metaphase) }\end{gathered}$ (metaphase)
PRC1
DNA
$\frac{2}{2}+3$

MUG

siNdc80

SICENP-E F low mis. high mis.
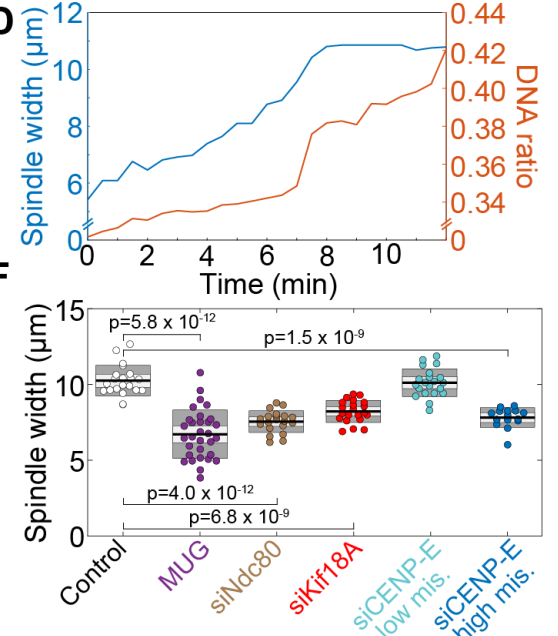
Fig. 4: Chromosomes separate microtubule bundles and increase spindle width.

a, Left, Vertical prometaphase rosette cross-section (single plane) in a HeLa-Kyoto BAC cell stably expressing PRC1-GFP, before (time 0 ) and after antiparallel bundle formation (4.5 minutes). Right, trajectories of all bundles from time 0 (free end of each curve) until 4.5 minutes (arrowheads), where the points between the end points were smoothed for better visibility and raw data are shown in Supplementary Video 5. Two large bundles visible at the top at time 0 are fixed in space (central points). Time interval between acquired images is 2.7 seconds. $\mathbf{b}$, Crosssection of a vertically oriented prometaphase rosette (single plane), showing DNA stained with SiR-DNA dye (left block) or PRC1-GFP (right block), as indicated, in a HeLa-Kyoto BAC cell stably expressing PRC1-GFP, at $\mathrm{t}=2 \mathrm{~min}$ (magenta) and $\mathrm{t}=4.5 \mathrm{~min}$ (green). Individual images are shown in white at the right of the merged images. Encircled areas marked "new" denote the chromosomes that arrived to the spindle midplane between $\mathrm{t}=2 \mathrm{~min}$ and $\mathrm{t}=4.5 \mathrm{~min}$, arrows denote the movement of DNA (left) or PRC1 bundles (right). $\mathbf{c}$, Time-lapse images of a horizontally oriented spindle in a HeLa-Kyoto BAC cell stably expressing PRC1-GFP (green), and DNA stained with SiR-DNA (magenta) starting with early prometaphase. Sum of 41 planes is shown. d, Spindle width and proportion of DNA at the metaphase plate over time for the cell shown in c. e, Live-cell images of metaphase spindles in HeLa-Kyoto BAC cells stably expressing PRC1-GFP (green), and DNA stained with SiR-DNA (magenta), treated as indicated. Maximal projections of 41 planes are shown. f, Spindle width for the indicated treatments. The black line shows the mean; the light and dark grey areas mark 95\% confidence interval on the mean and standard deviation, respectively. One-way ANOVA test showed significant difference between group means, p-values for significant differences from control are shown. Number of cells was 15-31 per group. Data for Kif18A siRNA are replotted from ${ }^{77}$. All scale bars, $1 \mu \mathrm{m}$.

\section{Theoretical model of bundle formation}

To understand the physics of bundle formation, we develop a theoretical model that allows us to identify the conditions that lead to the formation of multiple bundles, each bound to a kinetochore. We aim for a minimal model that includes key interactions between microtubules, kinetochores, chromosomes, and crosslinkers. As these interactions are complex, a minimal model is an optimal tool to understand the interplay between these multiple components and their roles in bundle formation, and to strengthen the hypotheses by comparison with experiments.

In our one-dimensional approach, representing the rim of the spindle midplane, we describe kinetochores as discrete points and explore the spatial distribution of microtubules (Fig. 5a). Kinetochores are considered as attractive points for microtubules, where multiple microtubules can bind and form a bundle. Microtubules experience mutual local attraction due to crosslinkers and inter-microtubule repulsion due to excluded volume effects. Finally, steric interactions between chromosomes and microtubules are described as a nonlocal intermicrotubule repulsion. A description of the model is given in Methods. 
The solutions of the model show that kinetochores play an important role in the

382 formation of microtubule bundles, with peaks of microtubule density at their positions (Fig.

383 5b). These peaks increase for an increasing kinetochore-microtubule attraction, which is

384 accompanied by a decrease of the microtubule density between the kinetochores. A decrease

385 in this attraction leads to an almost homogeneous microtubule distribution. A similar behavior

386 was observed in experiments, where depletion of CENP-E led to thinner bundles and its overexpression to thicker bundles (Fig. 2d and Fig. 3g,h). Thus, theory together with experiments suggest that microtubules respond to the kinetochore-microtubule attraction by rearranging themselves between a uniform distribution and pronounced bundles at kinetochores.

Even though kinetochores are major attraction sites, microtubule crosslinkers also affect the formation of bundles (Fig. 5c). An increase in the crosslinker concentration leads to more pronounced bundles at the kinetochores, showing a similar trend as the increase in kinetochore-microtubule attraction. In agreement with this theoretical result, experiments in which PRC1 was overexpressed showed excessively thick overlap microtubule bundles (Fig. 5d and Extended Data Fig. 5). Thus, microtubule crosslinkers act cooperatively with kinetochores in the formation of bundles.

Finally, we explored how the distance between kinetochores influences microtubule distributions. When we increased the distance, the bundles at the kinetochores remained the same, whereas the distribution of the microtubules between the kinetochores became predominantly uniform, with more microtubules at the midpoint than for smaller interkinetochore distance (Fig. 5e). We assume that the cases with larger and smaller distances correspond to the transition of the spindle from prometaphase to metaphase, because during

404 this transition increasingly more kinetochores arrive to the spindle midplane and thus their 405 distance decreases. Indeed, we observed in experiments that in early prometaphase the 406 microtubule distribution was roughly uniform, whereas at a later phase deeper valleys between 407 the peaks appeared (Fig. 1e,f). Taken together, these results support our main hypothesis that 408 the attractive interactions due to kinetochores and crosslinkers drive the formation of microtubule bundles. 
A

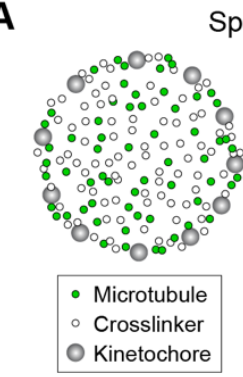

C

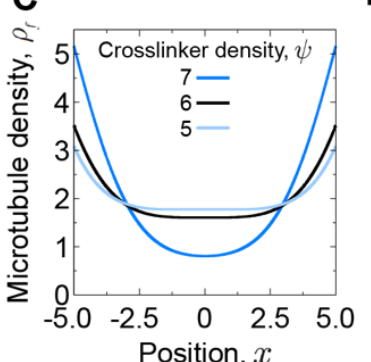

Figure 5

pindle midplane

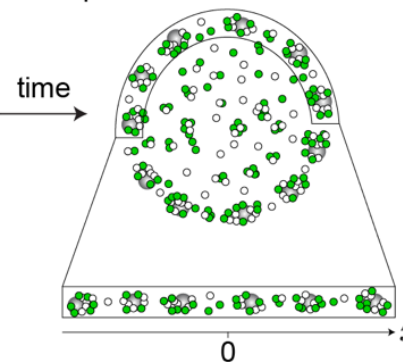

D

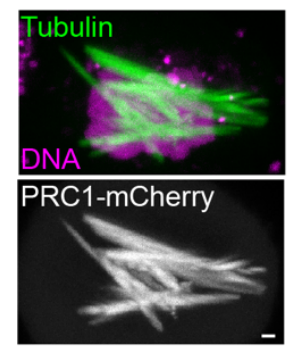

B

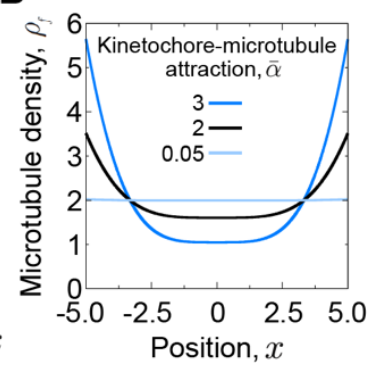

E

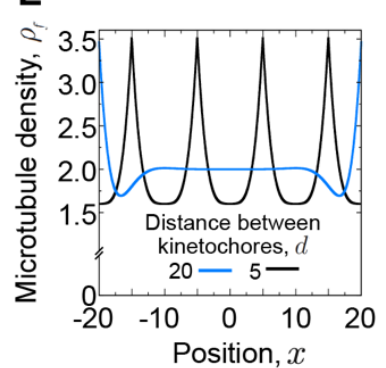

Fig. 5: Theoretical model of bundle formation.

a, Schematic representation of the model. Kinetochores (grey) are equally spaced along the edge of the spindle midplane. Microtubules (green) and crosslinkers (white) are initially uniformly distributed and gather into bundles over time. $\mathbf{b}$, Steady-state microtubule density, $\rho_{f}$, for 3 values of the kinetochore-microtubule attraction, $\bar{\alpha}$, as indicated. Other parameters are: $\psi=6, d=5, \rho_{b}=2, \kappa=5$ (all parameters are dimensionless, see Methods). The kinetochores are positioned at $x= \pm d$. c, Steady-state microtubule density, $\rho_{f}$, for 3 values of the crosslinker density, $\psi$, as indicated; $\bar{\alpha}=2, d=5, \rho_{b}=2, \kappa=5$. d, Spindle in a HeLa-TDS cell with overexpressed PRC1. Top: microtubules (SiR-tubulin, green) and DNA (Hoechst 33342, magenta), bottom: PRC1-mCherry (white). Scale bar, $1 \mu \mathrm{m}$. e, Steady-state microtubule density, $\rho_{f}$, for 2 values of the distance between kinetochores, $d$, as indicated; $\bar{\alpha}=2, \psi=6, \rho_{b}=2, \kappa=5$.

Functional role of PRC1-labeled bundles in chromosome segregation fidelity

426 Finally, we addressed the role of overlap bundles in chromosome segregation fidelity. It has been shown that in prometaphase microtubules adjacent to the centromeres stimulate kinetochore phosphorylation and thus correction of erroneous kinetochore-microtubule attachments through microtubule binding of Borealin, a component of the Chromosome

430 Passenger Complex ${ }^{71}$. We hypothesized that PRC1-bound overlap bundles are required for error correction, in a manner that Aurora B not only promotes the formation of these bundles (Fig. 2), but also uses them as tracks for the movement towards kinetochore targets to correct 433 the wrong attachments (Fig. 6a). This hypothesis predicts that reduction of overlap 
434 microtubules should lead to a decrease in outer kinetochore phosphorylation and an increase in

435 chromosome segregation errors.

436 To test these predictions, we reduced the overlap bundles by depleting PRC1, as this is

437 the most specific way to reduce these bundles known so far, which reduces overlap bundle

438 microtubules by about $50 \%$ without affecting kinetochore fibers ${ }^{22}$. We checked the

439 phosphorylation of serine 7 (Ser7) of CENP-A, a known Aurora B substrate ${ }^{78}$, and found a

440 significant drop in phosphorylation after PRC1 depletion (Fig. 6b,c), which suggests that

441 PRC1-crosslinked overlap microtubules promote kinetochore phosphorylation.

442 To assess the role of overlap bundles in chromosome segregation fidelity, we first 443 analyzed lagging kinetochores in anaphase and found that PRC1 depletion caused a small 444 increase in lagging kinetochores over control cells, from 1\% to 3.5\% (Fig. 6d,f; see Extended 445 Data Fig. 4 for depletion efficiency $)^{22}$. To directly examine the function of overlap bundles in 446 the correction of incorrect kinetochore attachments, we treated the cells with monastrol to block 447 the spindles in a monopolar state with high incidence of syntelic attachments, where both sister 448 kinetochores are attached to the same spindle pole ${ }^{15}$, and washed out monastrol after 40-60 449 minutes to allow for spindle assembly (Fig. 6d). Immunofluorescence analysis of spindles in 450 anaphase showed that monastrol-treated cells that were not depleted of PRC1 had only $2.3 \%$ 451 (Fig. 6f), indicating that error correction operates in cells with proper overlap bundles. In 452 contrast, monastrol-treated cells that were depleted of PRC1 had a significantly higher 453 incidence of lagging kinetochores, 10.8\% (Fig. 6e,f), revealing a role of overlap bundles in 454 error correction. Taken together, these results suggest that PRC1-bound overlap bundles have 455 a function in correcting erroneous kinetochore-microtubule attachments by promoting Aurora 456 B-driven kinetochore phosphorylation. 
A

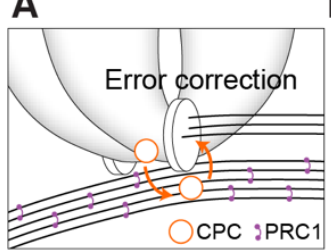

B

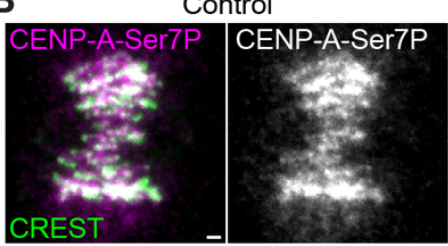

E

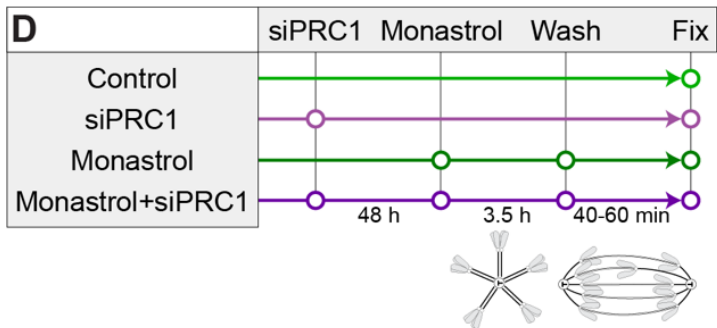

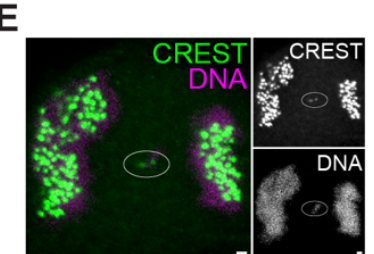

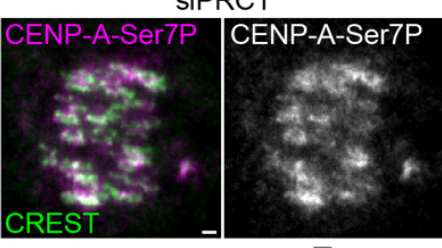

$\mathbf{F}$

459

460

Fig. 6: PRC1-bound overlap bundles promote chromosome segregation fidelity.

a, Scheme depicting the hypothesis that antiparallel overlaps help Aurora B to reach the kinetochore components, phosphorylate them and correct erroneous attachments. b. Images showing anti-CENP-A-Ser7P (magenta) and CREST (green) in control (left) and PRC1-depleted HeLa cells (right), merged channels and only anti-CENP-ASer7P in white are shown. $\mathbf{c}$, Ratio between anti-CENP-A-Ser7P and CREST in control $(\mathrm{n}=36)$ and PRC1-depleted cells $(n=33)$, from 3 immunostaining experiments. $\mathbf{d}$, Scheme of the protocol in which monastrol washout is used to increases erroneous attachments in prometaphase. e, Lagging chromosome (encircled) in anaphase in a PRC1depleted HeLa cell after monastrol washout. Left, merged image of CREST (green) and DNA (magenta). Right, individual channels (white). f, Frequency of lagging kinetochores in control (3 anaphases with lagging kinetochores / 299 total anaphases), PRC1 siRNA (8/231), monastrol washout (7/298), and PRC1siRNA+monatrol washout (28/259) from 3 immunostaining experiments per condition. One-way ANOVA test showed significant difference between group means, p-values are shown. The cell line used in this figure was unlabeled HeLa-TDS. All scale bars, $1 \mu \mathrm{m}$.

\section{DISCUSSION}

Kinetochore- and chromosome-driven transition from a dilute microtubule array to bundles

By combining STED microscopy, a live-cell bundling assay and theory, we have shown that spindle assembly occurs through a transition in which the initially uniform microtubule array is remodeled into bundles (Fig. 7a-c). Our experiments suggest that the temporal evolution of the bundles starts with the initial dilute network of antiparallel overlaps connecting the spindle poles, which relies on augmin-mediated microtubule nucleation together with the crosslinking 
activity of PRC1 and possibly other crosslinkers. When a kinetochore simultaneously attaches to the wall of several of these microtubules via CENP-E, this promotes further bundling of increasingly more microtubules by crosslinkers. While the chromosomes progressively congress to the spindle equator, they crowd there and thus move together with the attached bundles away from each other within the equatorial plane. As a result, the spindle cross-section widens and becomes uniformly filled with bundles and the attached chromosomes, and the spindle attains its metaphase shape.

We propose several competing mechanisms that are in action during transition to bundles. There are two attractive and two repulsive effects between the microtubules. Microtubules get attracted to neighboring microtubules via the crosslinkers (Fig. 7a). This process is facilitated locally by kinetochores because they attach to several adjacent microtubules, which brings the microtubules close to one another and promotes their bundling by crosslinkers (Fig. 7a). Microtubules experience local repulsion from neighbors due to excluded volume effects, and a non-local repulsion due to chromosomes whose crowding at the spindle midplane moves the attached bundles away from each other (Fig. 7b). This nonlocal repulsion helps to prevent all bundles from ending up as a single thick bundle. A bundling phase transition has been studied in theoretical models of cytoskeleton networks ${ }^{79,80}$, but here we show that additional interactions such as those between microtubules and kinetochores are important because they promote bundling. Yet, given that our model is one-dimensional, extensions of the model that would include inter-microtubule orientation may bring about new features of bundle formation.

\section{Kinetochores promote overlap bundle formation}

Our experiments revealed that kinetochores actively promote the formation of antiparallel bundles in prometaphase. Kinetochores have been previously implicated in the formation of antiparallel bundles in different contexts, for example during meiosis I in mouse oocytes, where

512 PRC1 is localized to the kinetochore through interaction with Ndc80, and facilitates bundling 513 of antiparallel overlaps in vicinity of the kinetochore ${ }^{81}$. As we did not find PRC1 at the

514 kinetochore in human somatic cells, the molecular mechanism of bundle formation may differ 515 in these two systems, thought the biophysical mechanisms may be similar. In anaphase in $C$.

516 elegans zygotes, formation of antiparallel bundles of the central spindle requires the 517 kinetochore proteins KNL-1, BUB-1, CENP-F, and CLASP ${ }^{82}$. CENP-E, which we identified to be important for microtubule bundling in prometaphase, is also present in the central spindle 
519 in anaphase, with an increased bundling activity ${ }^{83,84}$. Thus, it would be interesting to explore

520 the relationship between the kinetochore-related mechanisms that promote the formation of

521 microtubule bundles in prometaphase and anaphase, as well as their evolutionary aspects

522 through comparison between different organisms.

523 We found a role of CENP-E, but not of Ndc80 and dynein, in overlap bundle formation.

524 CENP-E, which is located in the fibrous corona connecting kinetochores to microtubules ${ }^{67}$, is

525 a very long and flexible molecule with a contour length of $230 \mathrm{~nm}^{85}$. This may allow multiple

526 CENP-E motors at an individual kinetochore to capture and bind laterally to several nearby

527 microtubules. In contrast, $\mathrm{Ndc} 80$ complex and cytoplasmic dynein are only 60 and $50 \mathrm{~nm}$ long

528 when extended, respectively ${ }^{86,87}$. This difference in length may explain the importance of

529 CENP-E in microtubule bundling, even though this it is not required for lateral attachment ${ }^{88}$.

530 Given that CENP-E interacts with $\mathrm{PRC1}^{83}$, their interaction may also be important for

531 microtubule bundling. In addition to PRC1, other proteins that bind to antiparallel

532 microtubules, such as Eg5, likely assist bundle formation. PRC1-interacting partners Kif4A

533 and MKLP1 may also play a role, similarly to their roles in anaphase ${ }^{27}$. These hypotheses

534 remain to be explored in future studies.

\section{Chromosomes separate the bundles and determine the spindle shape}

538 Bundling of microtubules by crosslinkers could in principle lead to bundling of most spindle microtubules into a single thick bundle. This is, however, not observed in animal spindles. In contrast, yeast spindles consist of a single microtubule bundle, which is likely due to a smaller number of microtubules, 40 in yeast versus 6000 in human spindles, and shorter spindles that prevent microtubule bending, $1.5 \mu \mathrm{m}$ long in yeast versus $10 \mu \mathrm{m}$ in human cells $^{38,89}$. We propose that in animal spindles chromosomes crowding at the metaphase plate, together with coupling the bundle with kinetochore during its formation, promotes bundle separation by driving outward movements of the bundles, which results in spindle widening. Several lines of evidence from our work support this notion. During prometaphase, as more and more chromosomes arrive at the spindle midplane and fill it, the spindle gets progressively wider. In agreement with this, spindles with perturbed chromosome alignment, where chromosome arrangement resembles more the prometaphase state rather than metaphase, are narrower. Examples studied here include depletion of Ndc80, Kif18A, and CENP-E, which have different mechanistic origin of chromosome misalignment ${ }^{74,75}$. Thus, it is unlikely that the observed spindle narrowing is due to perturbation of specific functions of these proteins, but rather due 
to misalignment itself. Another argument comes from mitosis with unreplicated genome, in which kinetochores interact with spindle microtubules but the DNA is dispersed throughout the cells ${ }^{10}$. As expected, this resulted in narrow spindles, but fully formed bundles due to the presence of kinetochores.

Previous work has shown that in HeLa cells, which have a variable number of chromosomes $^{90}$, spindle width increases with the number of chromosomes ${ }^{53}$. This result also supports the chromosome crowding hypothesis. Finally, a striking example comes from the finding that a single microtubule bundle connects the spindle poles with chromosomes lying along the sides upon expression of a kinase-inactive Aurora $\mathrm{B}^{91}$, in agreement with our hypothesis.

PRC1-linked overlap microtubules promote chromosome segregation fidelity

We have shown that PRC1-bound overlap bundles are required for the correction of synthelic kinetochore-microtubule attachments after spindle assembly from a monopole state. PRC1 depletion, which reduces the number of microtubules in the overalp bundles, resulted not only in an increase in lagging kinetochores, but also in decreased kinetochore phosphorylation. By combing these results with a previous work showing that Borealin binding to microtubules stimulates kinetochore phosphorylation and correction of erroneous kinetochore-microtubule attachments $^{71}$, we propose that Aurora B, being part of the Chromosome Passenger Complex together with Borealin, not only promotes the formation of PRC1-linked overlap bundles, but also uses them as tracks for the movement towards kinetochores to correct the wrong attachments. The molecular details of this PRC1-based error-correction mechanism remain an exciting topic for future studies.

In conclusion, our results reveal that a transition of microtubules from a loose array to bundles, dependent on kinetochores that serve as attraction sites and chromosomes that provide repulsion, promotes spindle assembly. In a broader sense, our study reveals general principles of organization of cytoskeletal filaments. The concepts of attraction sites as drivers of bundle formation may be relevant in other contexts, for example in the formation of actin bundles, where adherens junctions and focal adhesions may act as such sites. Overall, we expect that mechanisms similar to the ones identified here, though with different molecular players, act in a variety of cytoskeletal systems to generate specific filament arrangements required for their function. 
Bundle formation

Homogeneous network of antiparallel overalps

Kinetochores and PRC1 promote bundling

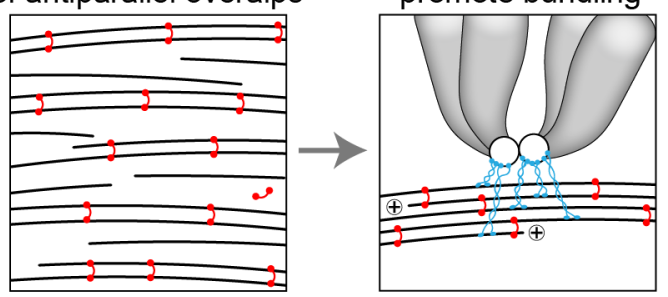

Figure 7

A

\section{C}

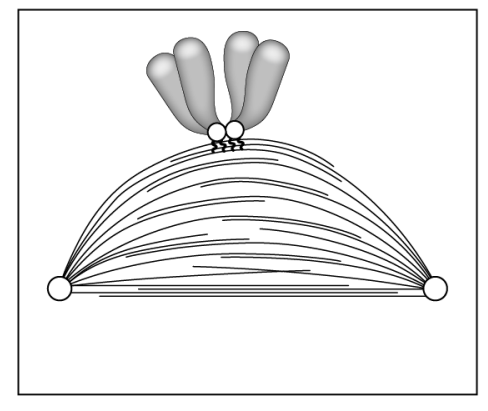

B

Chromosomes congress Chromosome crowding
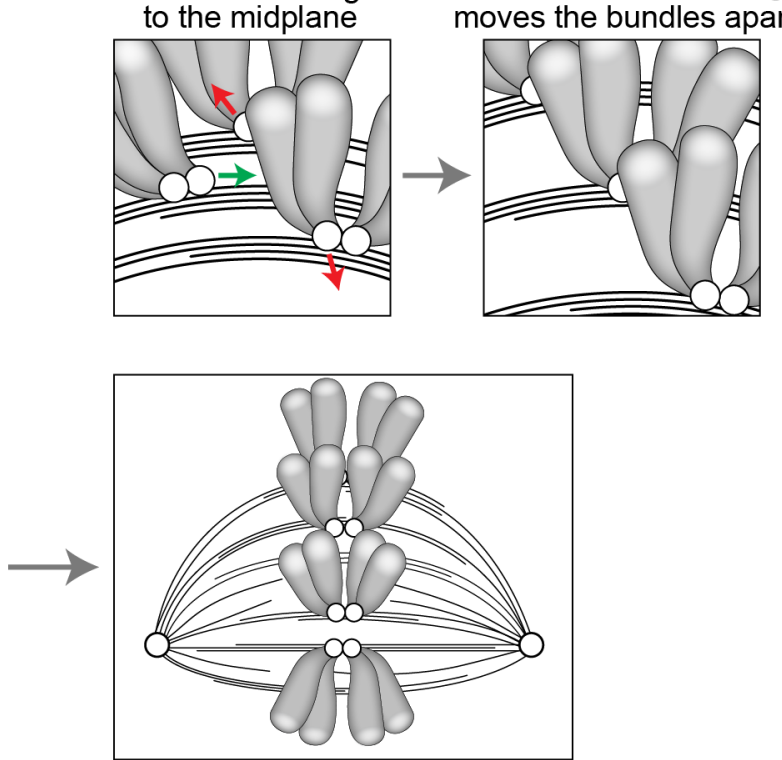

Fig. 7: Model for kinetochore driven overlap formation.

590 a, A loose microtubule network consisting of uniformly distributed antiparallel overlaps linked by PRC1 (red) undergoes a transition to tight bundles due to the microtubule-binding activity of kinetochores (CENP-E, blue). b, Bundles are separated (red arrows) during chromosome congression (green arrow) to the spindle midplane and steric effects of neighboring chromosome arms. $\mathbf{c}$, The processes depicted in $\mathbf{a}$ and $\mathbf{b}$ lead to a transition from the prometaphase rosette (left), with a loose network of microtubules and laterally attached chromosomes, to a more mature spindle in late prometaphase (right), with distinct and separated bundles. 


\section{REFERENCES}

1. Prosser, S. L. \& Pelletier, L. Mitotic spindle assembly in animal cells: A fine balancing act. Nat. Rev. Mol. Cell Biol. 18, 187-201 (2017).

2. McIntosh, J. R., Molodtsov, M. I. \& Ataullakhanov, F. I. Biophysics of mitosis. $Q$. Rev. Biophys. 45, 147-207 (2012).

3. Pavin, N. \& Tolić, I. M. Self-Organization and Forces in the Mitotic Spindle. Annu. Rev. Biophys. 45, 279-298 (2016).

4. McDonald, K. L., O’Toole, E. T., Mastronarde, D. N. \& McIntosh, J. R. Kinetochore microtubules in PTK cells. J. Cell Biol. 118, 369-383 (1992).

5. Cheeseman, I. M. \& Desai, A. Molecular architecture of the kinetochore-microtubule interface. Nat. Rev. Mol. Cell Biol. 9, 33-46 (2008).

6. Mastronarde, D. N., McDonald, K. L., Ding, R. \& McIntosh, J. R. Interpolar spindle microtubules in PTK cells. J. Cell Biol. 123, 1475-1489 (1993).

7. Mitchison, T. J. \& Kirschner, M. W. Properties of the Kinetochore In Vitro. I. Microtubule nucleation and tubulin binding. J. Cell Biol. 101, 755-765 (1985).

8. Rieder, C. L. \& Alexander, S. P. Kinetochores are transported poleward along a single astral microtubule during chromosome attachment to the spindle in newt lung cells. $J$. Cell Biol. 110, 81-95 (1990).

9. Wollman, R. et al. Efficient chromosome capture requires a bias in the 'search-andcapture' process during mitotic-spindle assembly. Curr. Biol. 15, 828-832 (2005).

10. O’Connell, C. B., Lončarek, J., Kaláb, P. \& Khodjakov, A. Relative contributions of chromatin and kinetochores to mitotic spindle assembly. J. Cell Biol. 187, 43-51 (2009).

11. Sikirzhytski, V. et al. Microtubules assemble near most kinetochores during early prometaphase in human cells. J. Cell Biol. 217, 2647-2659 (2018).

12. Renda, F. et al. Non-centrosomal microtubules at kinetochores promote rapid chromosome biorientation during mitosis in human cells. Curr. Biol. in press, (2022).

13. Sawin, K. E., Leguellec, K., Philippe, M. \& Mitchison, T. J. Mitotic spindle organization by a plus-end-directed microtubule motor. Nature 359, 540-543 (1992).

14. Kapitein, L. C. et al. The bipolar mitotic kinesin Eg5 moves on both microtubules that it crosslinks. Nature 435, (2005).

15. Kapoor, T. M., Mayer, T. U., Coughlin, M. L. \& Mitchison, T. J. Probing spindle assembly mechanisms with monastrol, a small molecule inhibitor of the mitotic kinesin, Eg5. J. Cell Biol. 150, (2000).

16. Blangy, A. et al. Phosphorylation by $\mathrm{p} 34 \mathrm{cdc} 2$ regulates spindle association of human $\mathrm{Eg} 5$, a kinesin-related motor essential for bipolar spindle formation in vivo. Cell 83, 1159-1169 (1995).

17. Kajtez, J. et al. Overlap microtubules link sister k-fibres and balance the forces on bioriented kinetochores. Nat. Commun. 7, (2016).

18. Milas, A. \& Tolić, I. Relaxation of interkinetochore tension after severing of a k-fiber depends on the length of the k-fiber stub. Matters Sel. (2016) doi:10.19185/matters.201603000025.

19. Elting, M. W., Prakash, M., Udy, D. B. \& Dumont, S. Mapping Load-Bearing in the Mammalian Spindle Reveals Local Kinetochore Fiber Anchorage that Provides Mechanical Isolation and Redundancy. Curr. Biol. 27, 2112-2122.e5 (2017).

20. Maiato, H., Gomes, A. M., Sousa, F. \& Barisic, M. Mechanisms of chromosome congression during mitosis. Biology (Basel). 6, 1-56 (2017).

21. Suresh, P., Long, A. F. \& Dumont, S. Microneedle manipulation of the mammalian spindle reveals specialized, short-lived reinforcement near chromosomes. Elife 9, 1-22 
661

662

663

664

665

666

667

668

669

670

671

672

673

674

675

676

677

678

679

680

681

682

683

684

685

686

687

688

689

690

691

692

693

694

695

696

697

(2020).

22. Jagrić, M., Risteski, P., Martinčić, J., Milas, A. \& Tolić, I. M. Optogenetic control of prc1 reveals its role in chromosome alignment on the spindle by overlap lengthdependent forces. Elife 10, 1-79 (2021).

23. Risteski, P. et al. Coordinated poleward flux of sister kinetochore fibers drives chromosome alignment. bioRxiv 2020.12.30.424837 (2021) doi:10.1101/2020.12.30.424837.

24. Brust-Mascher, I., Civelekoglu-Scholey, G., Kwon, M., Mogilner, A. \& Scholey, J. M. Model for anaphase B: Role of three mitotic motors in a switch from poleward flux to spindle elongation. Proc. Natl. Acad. Sci. U. S. A. 101, 15938-15943 (2004).

25. Brust-Mascher, I., Sommi, P., Cheerambathur, D. K. \& Scholey, J. M. Kinesin-5dependent Poleward Flux and Spindle Length Control in Drosophila Embryo Mitosis. Mol. Biol. Cell 20, 1749-1762 (2009).

26. Vukušić, K. et al. Microtubule Sliding within the Bridging Fiber Pushes Kinetochore Fibers Apart to Segregate Chromosomes. Dev. Cell 43, 11-23.e6 (2017).

27. Vukušić, K., Ponjavić, I., Buđa, R., Risteski, P. \& Tolić, I. M. Microtubule-sliding modules based on kinesins EG5 and PRC1-dependent KIF4A drive human spindle elongation. Dev. Cell 56, 1253-1267.e10 (2021).

28. Yu, C. H. et al. Central-spindle microtubules are strongly coupled to chromosomes during both anaphase A and anaphase B. Mol. Biol. Cell 30, 2503-2514 (2019).

29. Asthana, J., Cade, N. I., Normanno, D., Lim, W. M. \& Surrey, T. Gradual compaction of the central spindle decreases its dynamicity in PRC1 and $\mathrm{EB} 1$ gene-edited cells. Life Sci. Alliance 4, (2021).

30. Janson, M. E. et al. Crosslinkers and Motors Organize Dynamic Microtubules to Form Stable Bipolar Arrays in Fission Yeast. Cell 128, 357-368 (2007).

31. Kapitein, L. C. et al. Microtubule-Driven Multimerization Recruits ase1p onto Overlapping Microtubules. Curr. Biol. 18, 1713-1717 (2008).

32. Subramanian, R. et al. Insights into antiparallel microtubule crosslinking by PRC1, a conserved nonmotor microtubule binding protein. Cell 142, 433-443 (2010).

33. Bieling, P., Telley, I. A. \& Surrey, T. A minimal midzone protein module controls formation and length of antiparallel microtubule overlaps. Cell 142, 420-432 (2010).

34. Edelmaier, C. J. et al. Mechanisms of chromosome biorientation and bipolar spindle assembly analyzed by computational modeling. Elife 9, (2020).

35. Blackwell, R. et al. Physical determinants of bipolar mitotic spindle assembly and stability in fission yeast. Sci. $A d v$. 3, (2017).

36. Winters, L. et al. Pivoting of microtubules driven by minus-end-directed motors leads to spindle assembly. BMC Biol. 17, (2019).

37. Nédélec, F. Computer simulations reveal motor properties generating stable antiparallel microtubule interactions. J. Cell Biol. 158, (2002).

38. Kiewisz, R., Fabig, G., Conway, W., Needleman, D. \& Müller-Reichert, T. Threedimensional structure of the kinetochore-fibers in human mitotic spindles. bioRxiv 2021.11.13.468347 (2021).

39. Needleman, D. \& Dogic, Z. Active matter at the interface between materials science and cell biology. Nat. Rev. Mater. 2, 17048 (2017).

40. Roostalu, J., Rickman, J., Thomas, C., Nédélec, F. \& Surrey, T. Determinants of Polar versus Nematic Organization in Networks of Dynamic Microtubules and Mitotic Motors. Cell 175, (2018).

41. Wu, K. T. et al. Transition from turbulent to coherent flows in confined threedimensional active fluids. Science 355, (2017).

42. Hitt, A. L., Cross, A. R. \& Williams, R. C. Microtubule solutions display nematic 
liquid crystalline structure. J. Biol. Chem. 265, 1639-1647 (1990).

43. Kierfeld, J., Kühne, T. \& Lipowsky, R. Discontinuous unbinding transitions of filament bundles. Phys. Rev. Lett. 95, 1-4 (2005).

44. Huisman, B. A. H., Bolhuis, P. G. \& Fasolino, A. Phase transition to bundles of flexible supramolecular polymers. Phys. Rev. Lett. 100, (2008).

45. Borukhov, I., Bruinsma, R. F., Gelbart, W. M. \& Liu, A. J. Structural polymorphism of the cytoskeleton: A model of linker-assisted filament aggregation. Proc. Natl. Acad. Sci. U.S. A. 102, (2005).

46. Kachan, D., Müller, K. W., Wall, W. A. \& Levine, A. J. Discontinuous bundling transition in semiflexible polymer networks induced by Casimir interactions. Phys. Rev. E 94, (2016).

47. Maxian, O., Donev, A. \& Mogilner, A. Interplay between Brownian motion and crosslinking kinetics controls bundling dynamics in actin networks. bioRxiv 2021.09.17.460819 (2021) doi:https://doi.org/10.1101/2021.09.17.460819.

48. Huang, B., Babcock, H. \& Zhuang, X. Breaking the diffraction barrier: Superresolution imaging of cells. Cell 143, 1047-1058 (2010).

49. Bolzer, A. et al. Three-dimensional maps of all chromosomes in human male fibroblast nuclei and prometaphase rosettes. PLoS Biol. 3, 0826-0842 (2005).

50. Itoh, G. et al. Lateral attachment of kinetochores to microtubules is enriched in prometaphase rosette and facilitates chromosome alignment and bi-orientation establishment. Sci. Rep. 8, 1-18 (2018).

51. Goshima, G., Mayer, M., Zhang, N., Stuurman, N. \& Vale, R. D. Augmin: A protein complex required for centrosome-independent microtubule generation within the spindle. J. Cell Biol. 181, 421-429 (2008).

52. Uehara, R. et al. The augmin complex plays a critical role in spindle microtubule generation for mitotic progression and cytokinesis in human cells. Proc. Natl. Acad. Sci. U. S. A. 106, 6998-7003 (2009).

53. Polak, B., Risteski, P., Lesjak, S. \& Tolić, I. M. PRC 1-labeled microtubule bundles and kinetochore pairs show one-to-one association in metaphase. EMBO Rep. 18, $217-$ 230 (2017).

54. O'Toole, E., Morphew, M. \& Richard McIntosh, J. Electron tomography reveals aspects of spindle structure important for mechanical stability at metaphase. Mol. Biol. Cell 31, 184-195 (2020).

55. Nagele, R., Freeman, T., Mcmorrow, L. \& Lee, H. Precise spatial positioning of chromosomes during prometaphase: evidence for chromosomal order. Science $\mathbf{2 7 0}$, 1831-1835 (1995).

56. Chaly, N. \& Brown, D. L. The prometaphase configuration and chromosome order in early mitosis. J. Cell Sci. 91, 325-335 (1988).

57. DeLuca, J. G. et al. Kinetochore Microtubule Dynamics and Attachment Stability Are Regulated by Hec1. Cell 127, 969-982 (2006).

58. Welburn, J. P. I. et al. Aurora B Phosphorylates Spatially Distinct Targets to Differentially Regulate the Kinetochore-Microtubule Interface. Mol. Cell 38, 383-392 (2010).

59. Shrestha, R. L. et al. Aurora-B kinase pathway controls the lateral to end-on conversion of kinetochore-microtubule attachments in human cells. Nat. Commun. 8, 1-12 (2017).

60. Mortlock, A. A. et al. Discovery, synthesis, and in vivo activity of a new class of pyrazoloquinazolines as selective inhibitors of aurora B kinase. J. Med. Chem. 50, 2213-2224 (2007).

61. Dennis, M. et al. Phase i study of the Aurora B kinase inhibitor barasertib (AZD1152) 
to assess the pharmacokinetics, metabolism and excretion in patients with acute myeloid leukemia. Cancer Chemother. Pharmacol. 70, 461-469 (2012).

62. Liu, D., Vader, G., Vromans, M. J. M., Lampson, M. A. \& Lens, S. M. A. Sensing chromosome bi-orientation by spatial separation of Aurora B kinase from kinetochore substrates. Science 323, 1350-1353 (2009).

63. Kallio, M. J., McCleland, M. L., Todd Stukenberg, P. \& Gorbsky, G. J. Inhibition of Aurora B kinase blocks chromosome segregation, overrides the spindle checkpoint, and perturbs microtubule dynamics in mitosis. Curr. Biol. 12, 900-905 (2002).

64. Wang, E., Ballister, E. R. \& Lampson, M. A. Aurora B dynamics at centromeres create a diffusion-based phosphorylation gradient. J. Cell Biol. 194, 539-549 (2011).

65. Kim, Y., Holland, A. J., Lan, W. \& Cleveland, D. W. Aurora kinases and protein phosphatase 1 mediate chromosome congression through regulation of CENP-E. Cell 142, 444-455 (2010).

66. Cheeseman, I. M., Chappie, J. S., Wilson-Kubalek, E. M. \& Desai, A. The Conserved KMN Network Constitutes the Core Microtubule-Binding Site of the Kinetochore. Cell 127, 983-997 (2006).

67. Yao, X., Anderson, K. L. \& Cleveland, D. W. The microtubule-dependent motor centromere-associated protein E (CENP- E) is an integral component of kinetochore corona fibers that link centromeres to spindle microtubules. J. Cell Biol. 139, 435-447 (1997).

68. Qian, X. et al. Discovery of the first potent and selective inhibitor of centromereassociated protein E: GSK923295. ACS Med. Chem. Lett. 1, 30-34 (2010).

69. Griffis, E. R., Stuurman, N. \& Vale, R. D. Spindly, a novel protein essential for silencing the spindle assembly checkpoint, recruits dynein to the kinetochore. J. Cell Biol. 177, 1005-1015 (2007).

70. Ciferri, C. et al. Implications for Kinetochore-Microtubule Attachment from the Structure of an Engineered Ndc80 Complex. Cell 133, 427-439 (2008).

71. Trivedi, P. et al. The binding of Borealin to microtubules underlies a tension independent kinetochore-microtubule error correction pathway. Nat. Commun. 10, (2019).

72. Wise, D. A. \& Brinkley, B. R. Mitosis in cells with unreplicated genomes (MUGs): Spindle assembly and behavior of centromere fragments. Cell Motil. Cytoskeleton 36, (1997).

73. Martin-Lluesma, S., Stucke, V. M. \& Nigg, E. A. Role of Hec1 in spindle checkpoint signaling and kinetochore recruitment of Mad1/Mad2. Science 297, 2267-2270 (2002).

74. DeLuca, J. G., Moree, B., Hickey, J. M., Kilmartin, J. V. \& Salmon, E. D. hNuf2 inhibition blocks stable kinetochore-microtubule attachment and induces mitotic cell death in HeLa cells. J. Cell Biol. 159, (2002).

75. Stumpff, J., von Dassow, G., Wagenbach, M., Asbury, C. \& Wordeman, L. The Kinesin-8 Motor Kif18A Suppresses Kinetochore Movements to Control Mitotic Chromosome Alignment. Dev. Cell 14, 252-262 (2008).

76. Mayr, M. I. et al. The Human Kinesin Kif18A Is a Motile Microtubule Depolymerase Essential for Chromosome Congression. Curr. Biol. 17, (2007).

77. Trupinić, M. et al. Twist of the mitotic spindle culminates at anaphase onset and depends on microtubule-associated proteins along with external forces. bioRxiv (2020) doi:10.1101/2020.12.27.424486.

78. Zeitlin, S. G., Shelby, R. D. \& Sullivan, K. F. CENP-A is phosphorylated by Aurora B kinase and plays an unexpected role in completion of cytokinesis. J. Cell Biol. 155, 1147-1157 (2001).

79. Chandrasekaran, A., Upadhyaya, A. \& Papoian, G. A. Remarkable structural 
transformations of actin bundles are driven by their initial polarity, motor activity, crosslinking, and filament treadmilling. PLoS Comput. Biol. 15, (2019).

80. Weirich, K. L. et al. Liquid behavior of cross-linked actin bundles. Proc. Natl. Acad. Sci. U. S. A. 114, 2131-2136 (2017).

81. Yoshida, S. et al. Prc1-rich kinetochores are required for error-free acentrosomal spindle bipolarization during meiosis I in mouse oocytes. Nat. Commun. 11, (2020).

82. Maton, G. et al. Kinetochore components are required for central spindle assembly. Nat. Cell Biol. 17, 697-705 (2015).

83. Liu, X. et al. Mitotic motor CENP-E cooperates with PRC1 in temporal control of central spindle assembly. J. Mol. Cell Biol. 12, 654-665 (2020).

84. Vitre, B. et al. Kinetochore-microtubule attachment throughout mitosis potentiated by the elongated stalk of the kinetochore kinesin CENP-E. Mol. Biol. Cell 25, 2272-2281 (2014).

85. Kim, Y., Heuser, J. E., Waterman, C. M. \& Cleveland, D. W. CENP-E combines a slow, processive motor and a flexible coiled coil to produce an essential motile kinetochore tether. J. Cell Biol. 181, 411-419 (2008).

86. Wei, R. R., Sorger, P. K. \& Harrison, S. C. Molecular organization of the Ndc80 complex, an essential kinetochore component. Proc. Natl. Acad. Sci. U. S. A. 102, 5363-5367 (2005).

87. Vallee, R. B., Wall, J. S., Paschal, B. M. \& Shpetner, H. S. Microtubule-associated protein 1c from brain is a two-headed cytosolic dynein. Nature 332, 561-563 (1988).

88. Shrestha, R. L. \& Draviam, V. M. Lateral to end-on conversion of chromosomemicrotubule attachment requires kinesins cenp-e and MCAK. Curr. Biol. 23, 15141526 (2013).

89. Winey, M. et al. Three-dimensional ultrastructural analysis of the Saccharomyces cerevisiae mitotic spindle. J. Cell Biol. 129, 1601-1615 (1995).

90. Landry, J. J. M. et al. The genomic and transcriptomic landscape of a hela cell line. G3 Genes, Genomes, Genet. 3, 1213-1224 (2013).

91. Murata-Hori, M. \& Wang, Y. L. The kinase activity of aurora B is required for kinetochore-microtubule interactions during mitosis. Curr. Biol. 12, (2002).

92. Yang, J. et al. AZD1152, a novel and selective aurora B kinase inhibitor, induces growth arrest, apoptosis, and sensitization for tubulin depolymerizing agent or topoisomerase II inhibitor in human acute leukemia cells in vitro and in vivo. Blood 110, (2007).

93. Wood, K. W. et al. Antitumor activity of an allosteric inhibitor of centromereassociated protein-E. Proc. Natl. Acad. Sci. U. S. A. 107, 5839-5844 (2010).

94. Bennett, A. et al. Cenp-E inhibitor GSK923295: Novel synthetic route and use as a tool to generate aneuploidy. Oncotarget 6, 20921-20932 (2015).

95. Rizk, A. et al. Segmentation and quantification of subcellular structures in fluorescence microscopy images using Squassh. Nat. Protoc. 9, 586-596 (2014). 


\section{METHODS}

\section{Cell lines and culture}

843 Experiments were carried out using unlabeled human HeLa-TDS cells (also referred to as untransfected HeLa cells) from the High-Throughput Technology Development Studio (Max

845 Planck Institute of Molecular Cell Biology and Genetics, Dresden, Germany), HeLa-Kyoto 846 BAC lines stably expressing PRC1-GFP (also referred to as HeLa PRC1-GFP cells) are courtesy of Ina Poser and Tony Hyman (Max Planck Institute of Molecular Cell Biology and

848 Genetics, Dresden, Germany), and human hTERT-RPE1 (hTERT-immortalized retinal 849 pigment epithelium) cells stably expressing CENP-A-GFP and Centrin1-GFP, courtesy of 850 Alexey Khodjakov (Wadsworth Center, New York State Department of Health, Albany, NY, 851 USA). All cell lines were cultured in flasks in Dulbecco's Modified Eagle's Medium with $1 \mathrm{~g} / \mathrm{L}$ 852 D-glucose, pyruvate and L-glutamine (DMEM, Lonza, Basel, Switzerland), supplemented with 10\% (vol/vol) heat-inactivated Fetal Bovine Serum (FBS, Sigma Aldrich, St. Louis, MO, USA) and penicillin $(100 \mathrm{IU} / \mathrm{mL}) /$ streptomycin $(100 \mathrm{mg} / \mathrm{mL})$ solution (Lonza, Basel, Switzerland). For the selection of HeLa PRC1-GFP cell lines, $50 \mu \mathrm{g} / \mathrm{ml}$ geneticin was added to the medium (Life Technologies, Waltham, MA, USA). The cells were kept at $37^{\circ} \mathrm{C}$ and $5 \% \mathrm{CO}_{2}$ in a humidified incubator (Galaxy 170S $\mathrm{CO}_{2}$, Eppendorf, Hamburg, Germany) and regularly passaged at the confluence of $70-80 \%$.

\section{Sample preparation, siRNA, plasmids, and dyes}

At $80 \%$ confluence, DMEM medium was removed from the flask and cells were washed with $5 \mathrm{ml}$ of phosphate buffered saline (PBS). Then, $1 \mathrm{ml} \mathrm{1 \%}$ trypsin/ethylenediaminetetraacetic acid (EDTA, Biochrom AG, Berlin, Germany) was added to the flask and cells were incubated at $37^{\circ} \mathrm{C}$ and $5 \% \mathrm{CO}_{2}$ in a humidified incubator for 5 minutes. After the incubation, trypsin was blocked by adding $2 \mathrm{ml}$ of DMEM medium. For RNAi experiments, the cells were seeded to reach $60 \%$ confluence the next day and cultured on $35 \mathrm{~mm}$ uncoated dishes with $0.17 \mathrm{~mm}$ (\#1.5 coverglass) glass thickness (MatTek Corporation, Ashland, MA, USA) in $2 \mathrm{~mL}$ DMEM medium with the supplements described above. After one day of growth, cells were transfected with either targeting or non-targeting siRNA constructs which were diluted in OPTI-MEM medium (Life Technologies, Waltham, MA, USA) to a final concentration of $100 \mathrm{nM}$ in the medium with cells. All transfections were performed 48 hours prior to imaging using Lipofectamine RNAiMAX Reagent (Life Technologies, Waltham, MA, USA) according to the instructions provided by the manufacturer. After four hours of treatment, the medium was 
changed to the DMEM medium. The constructs used were human HAUS6 siRNA (L-01837201-0005, Dharmacon, Lafayette, CO, USA), human ON-TARGET plus SMART pool CENPE siRNA (L-003252-00-0010, Dharmacon, Lafayette, CO, USA), ON-TARGET human CCDC99 siRNA (L-016970-00-0010, Dharmacon, Lafayette, CO, USA), human Ndc80 siRNA (HA12977117-004; Merck, Darmstadt, Germany ), human ON-TARGET PRC1 siRNA (L-019491-00-0020, Dharmacon) and control siRNA (D-001810-10-05, Dharmacon, Lafayette, CO, USA).

For experiments with CENP-B-INCENP-GFP, unlabeled HeLa-TDS cells were transfected with CENP-B-INCENP-GFP (Addgene, plasmid \#45238), and for live-cell imaging also with mCherry-PRC1 plasmid provided by Casper C. Hoogenraad (Utrecht University, Utrecht, The Netherlands). $1 \times 10^{6}$ cells were transfected using Lipofectamine 3000 Reagent (L3000001, Life Technologies, Waltham, MA, US) with $1.5 \mu \mathrm{g}$ of plasmid DNA for both plasmids. For CENP-E overexpression experiments, unlabeled HeLa-TDS cells were transfected with $2 \mu \mathrm{g}$ of CENP-E-GFP plasmid (gift from Marin Barišić, Danish Cancer Society Research Center, Copenhagen, Denmark). To overexpress PRC1, unlabeled HeLaTDS cells were transfected with $5 \mu \mathrm{g}$ of mCherry-PRC1 plasmid. Transfection of both unlabeled HeLa-TDS and HeLa PRC1-GFP cells was performed 25-35 h before imaging.

In order to visualize microtubules, human hTERT-RPE1 cells stably expressing CENP-

892 A-GFP and Centrin1-GFP and unlabeled HeLa-TDS cells were stained to a final concentration of $100 \mathrm{nM}$ with a far-red silicon rhodamine (SiR)-tubulin-670 dye (Spirochrome, Stein am Rhein, Switzerland), 45 minutes to 2 hours prior to imaging. In order to avoid dye efflux, a broad-spectrum efflux pump inhibitor verapamil (Spirochrome, Stein am Rhein, Switzerland) was added at a final concentration of $0.5 \mu \mathrm{M}$ to the cells along with SiR-tubulin. For chromosome visualization, HeLa PRC1-GFP cells were stained with 100 nM SiR-DNA dye for 20 minutes to 2 hours prior to imaging. To identify the cells in mitosis by condensed chromosomes in the PRC1 overexpression experiment on unlabeled HeLa-TDS cells, $50 \mu \mathrm{L}$ of

900 NucBlue Live Ready Probes Reagent (Hoechst 33342, Invitrogen by Thermo Fisher Scientific, Waltham, MA, USA) was added to the dishes 1 min before imaging.

\section{Drug treatments}

904 The stock solution of the Aurora B kinase inhibitor Barasertib (AZD1152-HQPA,

905 Selleckchem, Munich, Germany) was prepared in Dimethyl sulfoxide (DMSO) to a final 906 concentration of $1 \mathrm{mM}$. The working solution was prepared in DMEM at $600 \mathrm{nM}$ and at the 907 time of treatment, the working solution was added to cells at 1:1 volume ratio to obtain a final 
908

909

910

concentration of $300 \mathrm{nM}$; IC50 is $0.35 \mathrm{nM}$ for this compound ${ }^{92}$. Barasertib was added at early prometaphase rosette in HeLa PRC1-GFP cell line. Appearance of predominantly prometaphase-like spindles ${ }^{63}$ in the sample imaged 1 hour post-treatment confirmed the effect of Barasertib on Aurora B.

The stock solution of the CENP-E inhibitor GSK-923295 (MedChemExpress, Monmouth Junction, NJ, USA) was prepared in DMSO at a final concentration of $8 \mathrm{mM}$. The working solution was prepared in DMEM at $200 \mathrm{nM}$. At the time of treatment, the working solution was added to cells at 1:1 volume ratio to obtain a final concentration of $100 \mathrm{nM}$; IC50 value of compound is $3.2 \mathrm{nM}^{93}$. GSK-923295 was added at early prometaphase rosette in HeLa PRC1-GFP cell line immediately before the start of imaging, and 2 hours before imaging in hTERT-RPE1 cell line expressing CenpA-GFP and Centrin1-GFP. Appearance of spindles blocked in prometaphase with a fraction of kinetochores trapped around polar region of the spindle at 30 minutes post-treatment ${ }^{94}$ confirmed the effect of GSK-923295. For hTERT-RPE1 cells, mock treatment was the same concentration of DMSO that was used for preparation of the inhibitors.

Nocodazole (HY-13520, MedChemExpress, Monmouth Junction, NJ, USA) working solution was prepared in DMEM at $2 \mu \mathrm{M}$. At the time of treatment, the working solution was added to cells at $1: 1$ volume ratio to obtain a final concentration of $1 \mu \mathrm{M}$. Nocodazole was added at early prometaphase rosette in HeLa PRC1-GFP cell line immediately before the start of imaging.

Monastrol (HY-101071A/CS-6183, MedChemExpress, Monmouth Junction, NJ, USA) working solution $(100 \mu \mathrm{M})$ was added in the dish with untransfected HeLa cells at a final concentration of $100 \mathrm{nM}$.

\section{Inducing mitosis with unreplicated genome (MUG)}

MUG is induced by incubation of cells in hydroxyurea and caffeine ${ }^{72}$, a gift from Anđela Horvat and Neda Slade (Laboratory for protein dynamics, Ruđer Bošković Institute, Zagreb, Croatia). The stock solutions of hydroxyurea and caffeine were prepared at a concentration of $175 \mathrm{mM}$ and $200 \mathrm{mM}$, respectively. The stock solution was diluted in DMEM to the final concentration of $2 \mathrm{mM}$ for hydroxyurea and $5 \mathrm{mM}$ for caffeine. The amount of $1 \times 10^{5} \mathrm{HeLa}$ PRC1-GFP cells were counted using the Improved Neubauer chamber (BRAND GMBH + CO $\mathrm{KG}$, Wertheim, Germany), seeded and cultured in $2 \mathrm{ml} \mathrm{DMEM}$ at $37^{\circ} \mathrm{C}$ and $5 \% \mathrm{CO}_{2}$ on $35 \mathrm{~mm}$ glass coverslip uncoated dishes with $0.17 \mathrm{~mm}$ (\#1.5 coverglass) glass thickness (MatTek Corporation, Ashland, MA, USA). After 12 hours, the medium was replaced with 2 mL DMEM 
containing $2 \mathrm{mM}$ hydroxyurea and incubated for 20 hours at $37^{\circ} \mathrm{C}$ and $5 \% \mathrm{CO}_{2}$. After 20 hours,

943 the medium was again replaced with a medium containing $2 \mathrm{mM}$ hydroxyurea and $5 \mathrm{mM}$

944 caffeine and the cells were again incubated at $37^{\circ} \mathrm{C}$ and $5 \% \mathrm{CO}_{2}$. The cells were imaged 18

945 hours later.

946

\section{Immunocytochemistry}

948 HeLa cells expressing PRC1-GFP and untransfected HeLa cells were grown on glass-bottom dishes (14 mm, No. 1.5, MatTek Corporation, Darmstadt, Germany) and fixed by $1 \mathrm{ml}$ of icecold methanol for $1 \mathrm{~min}$ at $-20^{\circ} \mathrm{C}$. Following fixation, cells were washed 3 times for 5 minutes with $1 \mathrm{ml}$ of PBS and permeabilized with $0.5 \%$ Triton-X-100 in water for 15 minutes at room temperature. To block unspecific binding, cells were incubated in $1 \mathrm{ml}$ of blocking buffer (1\% normal goat serum, NGS) for 1 hour at room temperature. Cells were then washed 3 times for 5 minutes with $1 \mathrm{ml}$ of PBS and incubated with $500 \mu 1$ of primary antibody solution overnight at $4^{\circ} \mathrm{C}$. The following primary antibodies were used: mouse monoclonal PRC1 (sc-376983, Santa Cruz Biotechnology), diluted 1:100, rat anti-alpha-tubulin YL1/2 (MA1-80017, Invitrogen, CA, SAD), diluted 1:500, mouse IgG monoclonal anti-GFP (Ref 11814460001, LOT42903200, Roche), diluted 1:100, rabbit anti-alpha-tubulin (SAB4500087, LOT 310379, Sigma Aldrich), diluted 1:500, human anti-CREST (15-235, Antibodic sinc), 1:100, Rabbit anti-CENP-E (C7488, Sigma Aldrich), diluted 1:100, Rabbit anti-Aurora B (ab239837, Abcam), diluted 1:100, anti-Hec1 (ab3613, Abcam), diluted 1:100, anti-Spindly (A301-354A, Biomolecules), diluted 1:100, anti-phospho-CENP-A (Ser7) (07-232, Sigma-Aldrich), diluted 1:500. After primary antibody, cells were washed in PBS and then incubated in $500 \mu \mathrm{L}$ of secondary antibody solution for $45 \mathrm{~min}$ at room temperature. The following secondary antibodies were used: donkey anti-mouse IgG Alexa Fluor 594, diluted 1:250 (ab150112, Abcam, Cambridge, UK), donkey anti-rabbit IgG Alexa Fluor 647 (ab150075, Abcam) and donkey anti-rat IgG Alexa Fluor 647 (ab150155, Abcam), Goat anti-human IgG 594 (ab96909, Abcam), all diluted 1:500. Finally, cells were washed with $1 \mathrm{~mL}$ of PBS, 3 times for $10 \mathrm{~min}$. This fixation was used for imaging HeLa PRC1-GFP cell line for siRNA silencing control, kinetochore and PRC1 visualization in vertical rosettes and for PRC1 visualization in different treatments (CENP-E overexpression, Aurora B relocalization) in untransfected HeLa cells.

To visualize alpha-tubulin in STED resolution in HeLa PRC1-GFP cell line, ice-cold methanol protocol was avoided because it destroyed the unstable fraction of microtubules.

974 Cells were washed with cell extraction buffer (CEB) and fixed by a microtubule-preserving 975 mixture of $3.2 \%$ paraformaldehyde (PFA) and $0.25 \%$ glutaraldehyde (GA) in microtubule- 
stabilizing PEM buffer (0.1 M PIPES, 0.001 M MgCl2 x 6 H2O, 0.001 M EDTA, 0.5 \% Triton$\mathrm{X}-100$ ) for $10 \mathrm{~min}$ at room temperature (Heuser and Kirschner, 1980). After fixation with PFA and GA, for quenching, cells were incubated in $1 \mathrm{~mL}$ of freshly prepared $0.1 \%$ borohydride in PBS for $7 \mathrm{~min}$ and after that in $1 \mathrm{~mL}$ of $100 \mathrm{mM} \mathrm{NH}_{4} \mathrm{Cl}$ and $100 \mathrm{mM}$ glycine in PBS for 10 min at room temperature. To block unspecific binding of antibodies, cells were incubated in $500 \mu \mathrm{L}$ blocking/permeabilization buffer ( $1 \%$ normal goat serum and $0.5 \%$ Triton-X-100 in water) for 2 hours at room temperature. Cells were then incubated in $500 \mu \mathrm{L}$ of primary antibody solution overnight at $4^{\circ} \mathrm{C}$. After the incubation with a primary antibody, cells were washed 3 times for 10 minutes with $1 \mathrm{ml}$ of PBS and then incubated with $500 \mu \mathrm{l}$ of secondary antibody for 2 hours at room temperature. This protocol was used in all experiments where tubulin is visualized in STED mode. In all fixations, DAPI $(1 \mu \mathrm{g} / \mathrm{mL})$ was used for chromosome visualization.

\section{Imaging}

990 STED microscope system (Abberior Instruments). STED microscopy was performed using the

991 Expert Line easy3D STED microscope system (Abberior Instruments) with a 60x/1.2 992 UPLSAPO 60XW water objective (Olympus), avalanche photodiode (APD) detector, and 993 Imspector software to acquire spindle cross-sections in horizontally and vertically oriented spindles in different stages of mitosis of HeLa cells expressing PRC1-GFP, immunostained for tubulin, with DNA stained by DAPI. STED images of tubulin were acquired in a single plane in the Alexa 594 channel with the excitation and depletion laser power at $35 \%$, and pixel size set $20 \mathrm{~nm}$. PRC1 and DNA were imaged in the same spindles in the confocal mode. Confocal mode was also used to image fixed prometaphase rosettes and metaphase spindles in untransfected and PRC1-GFP HeLa cells using eGFP and DAPI excitation lasers to visualize GFP and DAPI, respectively, or Alexa 488, 594, 647 excitation lasers depending on the secondary antibody. The laser power was $10 \%$, except for Alexa 647 where it was $5 \%$. Pixel size was $50 \mathrm{~nm}$ for prometaphase spindles and for imaging bridging fibers in metaphase, and $100 \mathrm{~nm}$ for metaphase spindles. Z-stacks of 41 focal planes, except in experiments on lagging kinetochores where the number of planes was 20 , were acquired with $0.5 \mu \mathrm{m}$ spacing to cover the whole spindle. For live cell imaging of vertical metaphase spindles in HeLa PRC1-GFP cell line, the middle plane was recognized as the plane in which the bundles appear as clear dots of PRC1-GFP and the spindle cross-section is filled with chromosomes. Four planes close to the spindle midplane were acquired, separated by $0.5 \mu \mathrm{m}$. Pixel size was $50 \mathrm{~nm}$. Laser power 
metaphase spindles was performed in HeLa PRC1-GFP cell line with Z-stacks of 41 planes using eGFP excitation light at 10\% laser power and 5\% laser power for SiR-DNA channel.

1012 Pixel size was $100 \mathrm{~nm}$. The bundling assay was applied for imaging of tubulin with SiR-tubulin

1013 dye in HeLa PRC1-GFP cell line. Time-lapse images of the spindle midplane were acquired

1014 using only SiR excitation laser with 8\% power. Time between repetitions was 1 minute and 1015 pixel size was $100 \mathrm{~nm}$.

Dragonfly spinning disk confocal microscope system (Andor Technology). To image

1017 the formation of PRC1-labeled bundles in HeLa cells expressing PRC1-GFP (bundling assay),

1018 confocal live-cell imaging was performed on a Dragonfly spinning disk microscope (Andor

1019 Technology, Belfast) using 63x/1.47 HC PL APO glycerol objective (Leica, Belfast) and Zyla

1020 4.2P scientific complementary metal oxide semiconductor (sCMOS) camera (Andor Technology). Images were acquired using Fusion software. During imaging, cells were maintained at $37^{\circ} \mathrm{C}$ and $5 \% \mathrm{CO}_{2}$ within a heating chamber (Okolab, Pozzuoli, NA, Italy). Only cells with vertically oriented spindles were imaged as follows: first a 41 plane Z-stack was acquired to cover the whole spindle using both $478 \mathrm{~nm}$ and $640 \mathrm{~nm}$ excitation light at a 10\% and 5\% laser power, respectively. Then a time-series of images was required using only 478 $\mathrm{nm}$ excitation light. Every repetition recorded 9 middle frames of the vertical spindle, forming a stack of 9 slices separated $0.5 \mu \mathrm{m}$. Time between repetitions was 5.4 seconds. After the timeseries, the second Z-stack was acquired, again using both excitation lasers. Total time-lapse movie was 9 minutes long. The same protocol was used for experiments with untreated cells, Haus6-depleted cells, and cells treated with Barasertib, GSK-923295, and nocodazole. For Ndc80, Haus6, and Spindly silencing control, a 41 plane Z-stack was acquired to cover the whole spindle using $478 \mathrm{~nm}, 620 \mathrm{~nm}$ and $408 \mathrm{~nm}$ excitation light at a 10\% for first two and 5\% laser power for DAPI channel. For all images pixel size was $100 \mathrm{~nm}$.

Opterra confocal microscope system (Bruker). For counting the number of bundles and for bundle trajectories, HeLa cells expressing PRC1-GFP and hTERT-RPE1 cells expressing CENP-A-GFP and Centrin1-GFP were imaged using Bruker Opterra Multipoint Scanning Confocal Microscope (Bruker Nano Surfaces, Middleton, WI, USA). The system was mounted on a Nikon Ti-E inverted microscope equipped with a Nikon CFI Plan Apo VC 100x/1.4 numerical aperture oil objective (Nikon, Tokyo, Japan). The system was controlled with the

1040 Prairie View Imaging Software (Bruker). During imaging, cells were maintained at $37^{\circ} \mathrm{C}$ in 1041 Okolab Cage Incubator (Okolab, Pozzuoli, NA, Italy). For optimal resolution and signal-to1042 noise ratio, $22 \mathrm{~nm}$ slit was used. For excitation of GFP, $488 \mathrm{~nm}$ diode laser line was used. The 1043 excitation light was separated from the emitted fluorescence by using Opterra Dichroic and 
1044 Barrier Filter Set 405/488/561/640. Images were captured with an Evolve 512 Delta Electron

1045 Multiplying Charge Coupled Device (EMCCD) Camera (Photometrics, Tucson, AZ, USA)

1046 using 150-200 ms exposure times. For counting the number of bundles, Z-stacks of 41 focal

1047 planes were acquired with $0.5 \mu \mathrm{m}$ spacing to cover the whole spindle. Horizontally oriented

1048 prometaphase spindles of HeLa PRC1-GFP cells with added SiR-DNA dye and hTERT-RPE1

1049 cells expressing CENP-A-GFP and Centrin1-GFP with added SiR-tubulin dye were filmed

1050 every 10 and 5 minutes, respectively. Prometaphase cells were recognized by non-congressed

1051 chromosomes and relatively small spindle size. To image the dynamics of PRC1-labeled

1052 bundles in HeLa cells expressing PRC1-GFP, confocal live-cell imaging was performed. Only

1053 cells with vertically oriented spindles were imaged as follows: first a 41 plane Z-stack was

1054 acquired to cover the whole spindle using both $488 \mathrm{~nm}$ and $640 \mathrm{~nm}$ excitation light. Then a

1055 time-series of images was required using only $488 \mathrm{~nm}$ excitation light. Every repetition recorded 9 middle frames of the vertically oriented spindle, forming a stack of 9 slices separated $0.5 \mu \mathrm{m}$. Time between repetitions was 2.7 seconds. After the time-series, a second Z-stack was acquired, again using both excitation lasers. Total time lapse movie was 4.5 minutes long. Laser powers were $10 \%$ and $5 \%$ for $488 \mathrm{~nm}$ and $640 \mathrm{~nm}$ excitation light, respectively.

1060

1061 Image processing and data analysis

1062 All images were analyzed in Fiji/ImageJ (National Institutes of Health, Bethesda, MD, USA).

1063 Raw images were used for quantification. Contrast was adjusted for clarity of presentation in 1064 the figures. MatLab (MathWorks, Natick, MA, USA) was used to create the plots, Rstudio (R 1065 Foundation for Statistical Computing, Vienna, Austria) to transform the horizontally oriented 1066 spindles into an end-on view. Figures were assembled in Adobe Illustrator CS5 (Adobe 1067 Systems, Mountain View, CA, USA).

1068 Bundling assay. For analysis of bundle formation, Squash plugin in Fiji was used in the

1069 PRC1-GFP channel of the middle plane of the spindle. Segmentation was performed using 1070 Squash ${ }^{95}$. In the main graphical user interface Squash window, we set the parameter for background subtraction option, entering the window edge lengths in units of 14 pixels. Object detection was performed over the entire image. The higher value of the regularization

1073 parameter of 0.1 was used to avoid the segmenting noise-induced small intensity peaks.

1074 Intensity values were normalized between 0 for the smallest value occurring in the image, and

10751 for the largest value. The sub-pixel segmentation was selected. The resolution of the

1076 segmentation was increased by an oversampling factor of 8 for 2D images. Local intensity estimation parameter was set to automatic. The Poisson model, recommended for confocal 
1078

1079

1080

1081

1082

1083

1084

1085

1086

1087

1088

1089

1090

1091

1092

1093

1094

1095

1096

1097

1098

1099

1100

1101

1102

1103

1104

1105

1106

1107

1108

1109

1110

1111

microscopes, was chosen. The following visualization options were selected: object intensities, number of objects and the mean object size in terms of area. Validation was performed by manually measuring object segmentation in the middle plane of a representative spindle.

Number of bundles. To count the bundles, horizontally oriented spindles were transformed into vertical (end-on) orientation using the code written in $\mathrm{R}$ programming language in R Studio (R Core Team., 2016). Before the transformation, the Z-stack of the spindle in a single channel was rotated in Fiji so that the spindle major axis was approximately parallel to the $\mathrm{x}$-axis. The aberrations caused by refractive index mismatch between immersion oil and aqueous sample were taken into account in the program by multiplying Z-step size by a correction factor of 0.81 to obtain the correct Z-distance. This factor corresponds to the ratio of the cell diameter in $\mathrm{y}$ and $\mathrm{z}$ direction, assuming that a mitotic cell is spherical. Bundles were counted on the sum intensity images of 5 central planes of the transformed spindle. The number of bundles was determined by counting the bright spots using Multi point tool in ImageJ.

Protein silencing. Analysis of the immunofluorescence signal was performed on the sum of all 41 planes. The territory of the spindle was encircled with a segmented line in Fiji and sum intensity was measured.

PRC1 intensity of early formed bundles in the prometaphase rosette. To measure the bundle intensity in vertically oriented early prometaphase rosette, bundles were encircled in Fiji and sum intensity was measured in only one plane of the cross section where the bundle was of highest intensity. Bundles with bound kinetochores were measured in the plane where the kinetochore was visible close to the bundle. The brightest bundles (usually two) that connect the spindle poles in a straight line were not measured because they are formed before nuclear envelope breakdown. Thus, only bundles in the prometaphase network were measured.

PRC1 intensity of bundles in metaphase. To measure the bundle intensity in vertically oriented metaphase spindles, each bundle was encircled in Fiji with a fixed-size circle $(p=0.61$ $\mu \mathrm{m}^{2}$ ) and mean intensity was measured in only one plane of the cross-section where DNA covered the whole cross-section. To calculate the PRC1 intensity of the bundle, mean intensity of PRC1 in the cytoplasm was subtracted from bundle PRC1 intensity. Furthermore, to correct for the differences in PRC1 expression in different cells, the PRC1 bundle intensity was divided by the mean intensity of PRC1 in the cytoplasm to get the normalized PRC1 intensity.

PRC1 intensity of the network in early prometaphase. To measure the network intensity in vertically oriented prometaphase spindles, the region excluding the brightest bundles (the ones formed before nuclear envelope breakdown) was encircled in Fiji and mean intensity was measured in only one plane. As for the PRC1 intensity of bundles, the mean intensity of PRC1 
1112 in the cytoplasm was subtracted from the network PRC1 intensity, and the result was divided

1113 by the mean intensity of PRC1 in the cytoplasm to get the normalized PRC1 intensity.

1114 Tubulin intensity of bridging fibers in metaphase. Intensity of bridging fibers was

1115 measured on metaphase spindles. A line was drawn along the bridging fiber using Line tool in

1116 Fiji, only the region beneath the kinetochore pair was measured. Intensity of tubulin was

1117 measured at the midpoint of the line intensity plot. Furthermore, to correct for the differences

1118 in tubulin signal in different cells, tubulin bundle intensity was divided by the mean intensity

1119 of tubulin in the cytoplasm to get the normalized tubulin intensity.

$1120 \quad$ PRC1 line intensity in metaphase. In HeLa PRC1-GFP cell line, a line was drawn from

1121 pole to pole in a metaphase spindle, which was recognized by fully aligned chromosomes in

1122 control cells. In treatments that cause impaired chromosome congression, spindles with most

1123 chromosomes aligned were chosen. Line intensity plot was made by subtracting the 1124 cytoplasmatic PRC1 signal and dividing by it, providing the normalized PRC1 intensity.

Aurora B-PRC1 correlation. Individual PRC1-labeled bridging fibers were encircled

with a circle that goes from the top edge of the bundle to the bottom edge, and the sum intensity was measured. The same-size circle was used to measure the sum intensity of the Aurora B signal on the associated centromere. Normalized intensity of PRC1 and Aurora B was obtained by subtracting the cytoplasmatic mean intensity of the respective protein and dividing by it.

Phosphorylation of outer kinetochore. Sum intensity of CREST and CENP-A signal was measured in prometaphase cells. The spindle was boxed in a fixed square that was same for all cells. The intensity ratio was expressed by dividing the CENP-A-Ser7P sum intensity by the CREST sum intensity.

1134 Spindle parameters. Spindle width and length were measured on horizontally oriented metaphase spindles using Line tool in Fiji.

Manual bundle tracking. The 2D trajectories of the bundles and their coordinates were tracked using Manual tracking tool to obtain bundle trajectories. The movement of all bundles was represented with respect to the brightest bundle(s) that appear before nuclear envelope breakdown and do not have chromosomes attached in early prometaphase, using Manual drift correction plugin in Fiji.

1141 DNA occupancy in the metaphase. Sum intensity of SiR-DNA signal from 41 planes 1142 was used for measurement of DNA occupancy in the equatorial region of the spindle. 1143 Proportion of DNA at the metaphase plate over time was measured as mean intensity of the 1144 DNA within the line covering the equatorial region divided by the intensity of all DNA in the 1145 cell. The line covering the equatorial region was defined in metaphase so that its width was 
1146 equal to the width of the metaphase plate and length equal to spindle width. The same line was

1147 used for all time frames.

1148 Lagging kinetochores were identified by the CREST signal together with the DNA

1149 signal in the central part of the spindle in anaphase, between the two segregated groups of

1150 kinetochores/chromosomes.

Statistical analysis and figure preparation. Graphs were generated in Matlab

1152 (MathWorks, Natick, MA, USA). Fiji was used to scale images and adjust brightness and

1153 contrast. Figures were assembled in Illustrator (Adobe Systems, Mountain View, CA, USA).

1154 Data are given as mean \pm s.e.m., unless otherwise stated. Means of two groups were compared

1155 by Student's t-test (two-tailed and two-sample unequal-variance), and means of more than two

1156 groups by one-way ANOVA test and Tukey's HSD post hoc test, $\mathrm{p}<0.05$ was considered

1157 statistically significant. The numbers of microtubule bundles, cells, and independent 1158 experiments are given in the figure panels.

1159 


\section{Theoretical model}

To describe the dynamical transition from array like distribution of microtubules to well organized multiple bundles during prometaphase, we consider both equilibrium and non equilibrium processes involving microtubules, crosslinking proteins, chromosomes, and kinetochores. The model we construct is placed in an one dimensional geometry with spatial coordinate $x$, taken from the part of the circumference of the disc that is obtained by taking a vertical cross section at the mid plane of a prometaphase spindle as shown in Fig 5A. Microtubules and crosslinkers are described as point like objects with densities $\tilde{\rho}$ and $\tilde{\psi}$, respectively. For simplicity, we assume that crosslinker have a uniform density.

In our model, we include interactions between microtubules, crosslinkers and kinetochores. We describe these interactions by constructing a Landau-Ginzburg free energy:

$$
F[\tilde{\rho}]=\int d \tilde{x}\left\{\frac{\tilde{w}}{4} \tilde{\rho}^{4}+\frac{\tilde{\kappa}}{2}\left(\frac{\partial \tilde{\rho}}{\partial \tilde{x}}\right)^{2}-\tilde{\zeta} \tilde{\rho} \tilde{\psi} \tilde{\rho}-\tilde{\alpha} \tilde{\rho}^{2} \delta(\tilde{x} \pm(2 n+1) \tilde{d})\right\} .
$$

In the first term we describe local microtubule repulsion as nonlinear interaction with strength $\tilde{w}$. The second term captures a non-local repulsion of strength $\tilde{\kappa}$, which is coming from interaction between chromosomes and microtubules. The third term describes an attractive interaction between two microtubules mediated by crosslinkers and with coupling constant $\tilde{\zeta}$. The last term represents attraction between kinetorchores and microtubules of strength $\tilde{\alpha}$. This local attraction is described by Dirac delta function. In our system, kinetochores are placed in regular intervals of given integer index $n$, forming lattice-like structure with spacing $2 \tilde{d}$.

Time evolution of microtubule density can be obtained by writing a conserved dynamics of $\tilde{\rho}$ by minimizing $F[\tilde{\rho}]$ and incorporating non equilibrium process of appearance and disappearance of microtubules in our region of interest. Rapid polymerization and depolymerization of microtubule filaments causes the processes of creation and annihilation of those filaments in the mid plane of the vertically oriented spindle. We assume that microtubules are created with a constant rate $\omega_{o n}$ and get disassembled with a rate $\omega_{\text {off }}$. Finally, we calculate how microtubule density changes in time $t$ as

$$
\frac{\partial \tilde{\rho}}{\partial t}=\tilde{w} \tilde{M}_{\rho} \frac{\partial^{2} \tilde{\rho}^{3}}{\partial \tilde{x}^{2}}-\tilde{\kappa} \tilde{M}_{\rho} \frac{\partial^{4} \tilde{\rho}}{\partial \tilde{x}^{4}}-2 \tilde{\zeta} \tilde{M}_{\rho} \tilde{\psi}_{\mathcal{M}} \tilde{M}_{\rho} \frac{\partial^{2} \tilde{\rho}}{\partial \tilde{x}^{2}}-2 \tilde{\alpha} \tilde{M}_{\rho} \frac{\partial^{2}}{\partial \tilde{x}^{2}}\{\tilde{\rho} \delta(\tilde{x} \pm(2 n+1) \tilde{d})\}+\omega_{o n} \tilde{\rho}_{b}-\omega_{o f f} \tilde{\rho}
$$

Here, $\tilde{\rho}_{b}$ denotes microtubule nucleation density in the cytoplasm and $\tilde{M}_{\rho}$ is microtubule mobility. Now, we proceed to make this time evolution equation dimensionless by using a length scale $x=\tilde{x}(\tilde{w})^{-\frac{1}{3}}$ and a time scale $\tau=t \omega_{\text {off }}$ and obtain,

$$
\frac{\partial \rho}{\partial \tau}=M_{\rho} \frac{\partial^{2} \rho^{3}}{\partial x^{2}}-\kappa M_{\rho} \frac{\partial^{4} \rho}{\partial x^{4}}-2 \zeta M_{\rho} \psi \frac{\partial^{2} \rho}{\partial x^{2}}-2 \bar{\alpha} M_{\rho} \frac{\partial^{2}}{\partial x^{2}}\{\rho \delta(x \pm(2 n+1) d)\}+\omega \rho_{b}-\rho,
$$

where $\rho=\tilde{\rho} \tilde{w}^{\frac{1}{3}}, \psi=\tilde{\psi} \tilde{w}^{\frac{1}{3}}, \zeta=\tilde{\zeta} \tilde{w}^{-\frac{2}{3}}, \kappa=\tilde{\kappa} \tilde{w}^{-1}, \bar{\alpha}=\tilde{\alpha} \tilde{w}^{-1}, d=\tilde{d} \tilde{w}^{-\frac{1}{3}}, \rho_{b}=\tilde{\rho}_{b} \tilde{w}^{\frac{1}{3}}, M_{\rho}=\tilde{M}_{\rho} \omega_{o f f}^{-1}$ and $\omega=\frac{\omega_{o n}}{\omega_{o f f}}$.

\section{A. Boundary conditions and steady state density profiles}

To study the kinetochore driven bundling of microtubules in the linear regime, we construct linearized steady state equation with steady state microtubule density $\rho_{f}$ from Eq.(2) by setting $\rho=\rho_{s}+\rho_{f}$, where $\rho_{s}=\frac{\omega_{o n}}{\omega_{o f f}} \rho_{b}$. The steady state equation with delta-function at $\pm d$ reads as

$$
M_{\rho}\left[\left(3 \rho_{s}^{2}-2 \zeta \psi\right) \frac{\partial^{2} \rho_{f}}{\partial x^{2}}-\kappa \frac{\partial^{4} \rho_{f}}{\partial x^{4}}\right]-\rho_{f}-2 \bar{\alpha} M_{\rho} \frac{\partial^{2}}{\partial x^{2}}\left\{\left(\rho_{s}+\rho_{f}\right) \delta(x \pm d)\right\}=0
$$


The last term in Eq.(3) is vanishing at all positions except at $\pm d$. Thus, we first solve Eq.(3) between boundary points by searching for solutions in the form $\rho_{f}(x)=\sum_{j=1}^{4} C_{j} e^{-i m_{j} x}$ and obtain

$$
m^{4}+\eta_{1} m^{2}+\eta_{2}=0
$$

where, $\eta_{1}=\frac{3 \rho_{s}^{2}-2 \zeta \psi}{2 \kappa}$ and $\eta_{2}=\frac{1}{\kappa M_{\rho}}$. Symmetry of Eq.(4) ensures the four solution $m_{1,2,3,4}$ can be written as $m_{3}=-m_{1}$ and $m_{4}=-m_{2}$ and consequently we obtain the density profile in steady state as

$$
\rho_{f}(x)=C_{1} e^{-i m_{1} x}+C_{2} e^{-i m_{2} x}+C_{3} e^{i m_{1} x}+C_{4} e^{i m_{2} x},
$$

$C_{1, . ., 4}$ being constants of integration. The structure of the system with two attractive $\delta$ functions at $\pm d$ ensures the existence of mirror symmetry $\rho_{f}(-x)=\rho_{f}(x)$ and Eq.(5) reduces to,

$$
\rho_{f}(x)=C_{1}\left(e^{i m_{1} x}+e^{-i m_{1} x}\right)+C_{2}\left(e^{i m_{2} x}+e^{-i m_{2} x}\right)
$$

Performing repeated integration of Eq.(3) and finally in the $\lim _{\epsilon \rightarrow 0}, \int_{d-\epsilon}^{d+\epsilon}$ around the $\delta$ function yields,

$$
\kappa\left[\rho_{f}^{\prime}(-d)-\rho_{f}^{\prime}(d)\right]+2 \bar{\alpha} \rho_{s}+2 \bar{\alpha} \rho_{f}(d)=0
$$

Here, we have used the translational invarience of the density $\rho(x+2 d)=\rho(x)$. Eq.(7) implies a discontinuous change in the first derivative of $\rho_{f}$ at $\pm d$. Similarly, between two boundaries, where the attractive interaction due to kinetochore is absent one obtains by integrating Eq.(3)

$$
\left[\frac{\partial^{3} \rho_{f}}{\partial x^{3}}\right]_{-d}^{d}-\eta_{1}\left[\frac{\partial \rho_{f}}{\partial x}\right]_{-d}^{d}+\eta_{2} \int_{-d}^{d} \rho_{f}(x) d x=0
$$

Eq.(6), Eq.(7), and Eq.(8) altogether give us the full density profiles depending on the nature of the roots $m_{1}$ and $m_{2}$.

When $m_{1}$ and $m_{2}$ are purely imaginary, we have

$$
\begin{aligned}
\rho_{f}(x) & =2 C_{1} \cosh \left(m_{1} x\right)+2 C_{2} \cosh \left(m_{2} x\right), \\
C_{1} & =\frac{\rho_{s}}{2\left[\frac{\kappa}{\bar{\alpha}} m_{1} \sinh \left(m_{1} d\right)-\cosh \left(m_{1} d\right)\right]-2 \frac{P_{1}}{Q_{1}}\left[\frac{\kappa}{\bar{\alpha}} m_{2} \sinh \left(m_{2} d\right)-\cosh \left(m_{2} d\right)\right]}, \\
C_{2} & =-\frac{\rho_{s}}{2 \frac{Q_{1}}{P_{1}}\left[\frac{\kappa}{\bar{\alpha}} m_{1} \sinh \left(m_{1} d\right)-\cosh \left(m_{1} d\right)\right]-2\left[\frac{\kappa}{\bar{\alpha}} m_{2} \sinh \left(m_{2} d\right)-\cosh \left(m_{2} d\right)\right]}
\end{aligned}
$$

with $P_{1}=\sinh \left(m_{1} d\right)\left\{m_{1}^{3}-m_{1} \eta_{1}+\frac{\eta_{2}}{m_{1}}\right\}$ and $Q_{1}=\sinh \left(m_{2} d\right)\left\{m_{2}^{3}-m_{2} \eta_{1}+\frac{\eta_{2}}{m_{2}}\right\}$.

As $m_{1}$ and $m_{2}$ become a pair of complex conjugate roots written as $m_{1}=a+i b$ and $m_{2}=a-i b$, we obtain a density profile given by

$$
\begin{aligned}
\rho_{f}(x) & =4 C \cosh (b x) \cos (a x)+4 \bar{C} \sinh (b x) \sin (a x), \\
C & =\frac{\rho_{s}}{4 \frac{P_{2}}{Q_{2}}\left[\beta_{2}-\frac{\kappa}{\bar{\alpha}}\left(b \alpha_{1}+a \beta_{1}\right)\right]-4\left[\alpha_{2}+\frac{\kappa}{\bar{\alpha}}\left(a \alpha_{1}-b \beta_{1}\right)\right]}, \\
\bar{C} & =-\frac{\rho_{s}}{4\left[\beta_{2}-\frac{\kappa}{\bar{\alpha}}\left(b \alpha_{1}+a \beta_{1}\right)\right]-4 \frac{Q_{2}}{P_{2}}\left[\alpha_{2}+\frac{\kappa}{\bar{\alpha}}\left(a \alpha_{1}-b \beta_{1}\right)\right]}
\end{aligned}
$$

where $C=C_{1}+C_{2}, \bar{C}=C_{1}-C_{2}, \alpha_{1}=\cosh (b d) \sin (a d), \beta_{1}=\sinh (b d) \cos (a d), \alpha_{2}=\cosh (b d) \cos (a d), \beta_{2}=$ $\sinh (b d) \sin (a d), P_{2}=\alpha_{1}\left\{a\left(a^{2}--3 b^{2}\right)\right\}+\beta_{1}\left\{b\left(b^{2}-3 a^{2}\right)\right\}+\eta_{1}\left(a \alpha_{1}-b \beta_{1}\right)+\frac{\eta_{2}}{a^{2}+b^{2}}\left(a \alpha_{1}+b \beta_{1}\right)$, and $Q_{2}=\alpha_{1}\left\{b\left(b^{2}-\right.\right.$ $\left.\left.3 a^{2}\right)\right\}-\beta_{1}\left\{a\left(a^{2}--3 b^{2}\right)\right\}-\eta_{1}\left(b \alpha_{1}+a \beta_{1}\right)+\frac{\eta_{2}}{a^{2}+b^{2}}\left(b \alpha_{1}-a \beta_{1}\right)$. 
For real $m_{1}$ and $m_{2}$ we have,

$$
\begin{aligned}
\rho_{f}(x) & =2 C_{1} \cos \left(m_{1} x\right)+2 C_{2} \cos \left(m_{2} x\right), \\
C_{1} & =\frac{\rho_{s}}{2\left[\frac{\kappa}{\bar{\alpha}} m_{1} \sin \left(m_{1} d\right)+\cosh \left(m_{1} d\right)\right]-2 \frac{P_{3}}{Q_{3}}\left[\frac{\kappa}{\bar{\alpha}} m_{2} \sin \left(m_{2} d\right)+\cos \left(m_{2} d\right)\right]}, \\
C_{2} & =-\frac{\rho_{s}}{2 \frac{Q_{3}}{P_{3}}\left[\frac{\kappa}{\bar{\alpha}} m_{1} \sin \left(m_{1} d\right)+\cos \left(m_{1} d\right)\right]-2\left[\frac{\kappa}{\bar{\alpha}} m_{2} \sin \left(m_{2} d\right)+\cos \left(m_{2} d\right)\right]},
\end{aligned}
$$

with $P_{3}=\sin \left(m_{1} d\right)\left\{m_{1}^{3}+m_{1} \eta_{1}+\frac{\eta_{2}}{m_{1}}\right\}$ and $Q_{3}=\sin \left(m_{2} d\right)\left\{m_{2}^{3}+m_{2} \eta_{1}+\frac{\eta_{2}}{m_{2}}\right\}$.

\section{ACKNOWLEDGEMENTS}

1161 We thank Ina Poser, Tony Hyman, Alexey Khodjakov, Marin Barišić, Casper Hoogenraad, and

1162 Neda Slade for cells, plasmids, and chemicals, Ivana Šarić for the drawings, and all members

1163 of Tolić and Pavin groups for helpful discussions. This work was funded by the European

1164 Research Council (ERC Synergy Grant, GA Number 855158, granted to I.M.T. and N.P.), the

1165 Croatian Science Foundation (HRZZ, project PZS-2019-02-7653 granted to I.M.T.), and

1166 projects co-financed by the Croatian Government and European Union through the European

1167 Regional Development Fund - the Competitiveness and Cohesion Operational Programme:

1168 IPSted (Grant KK.01.1.1.04.0057) and QuantiXLie Center of Excellence (grant

1169 KK.01.1.1.01.0004).

1170

1171

\section{AUTHOR CONTRIBUTIONS}

1172 J.M. performed and analyzed all experiments, except the experiments with lagging

1173 kinetochores and PRC1 and CENP-E overexpression, which were done by M.Ć. S.G.

1174 developed the theoretical model. I.M.T. and N.P. conceived the project and supervised the

1175 experimental and theoretical work, respectively. I.M.T., J.M., and N.P. wrote the paper with 1176 input from all authors.

1177

1178 COMPETING INTERESTS

1179 The authors declare no competing interests.

1180

1181 
bioRxiv preprint doi: https://doi.org/10.1101/2022.02.25.481924; this version posted February 25, 2022. The copyright holder for this

preprint (which was not certified by peer review) is the author/funder, who has granted bioRxiv a license to display the preprint in perpetuity. It is made available under aCC-BY-NC-ND 4.0 International license.

1183

Supplementary Figure 1
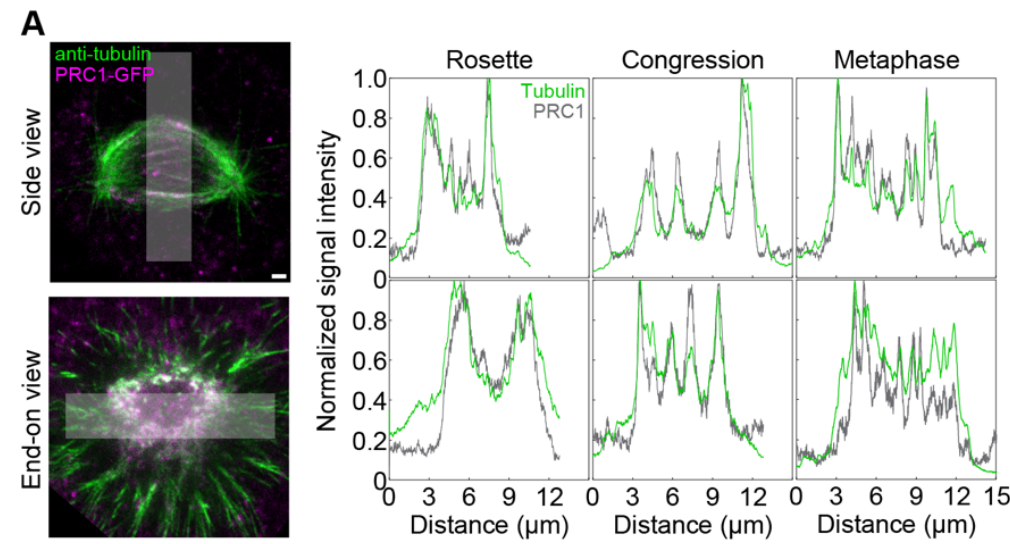

B

C
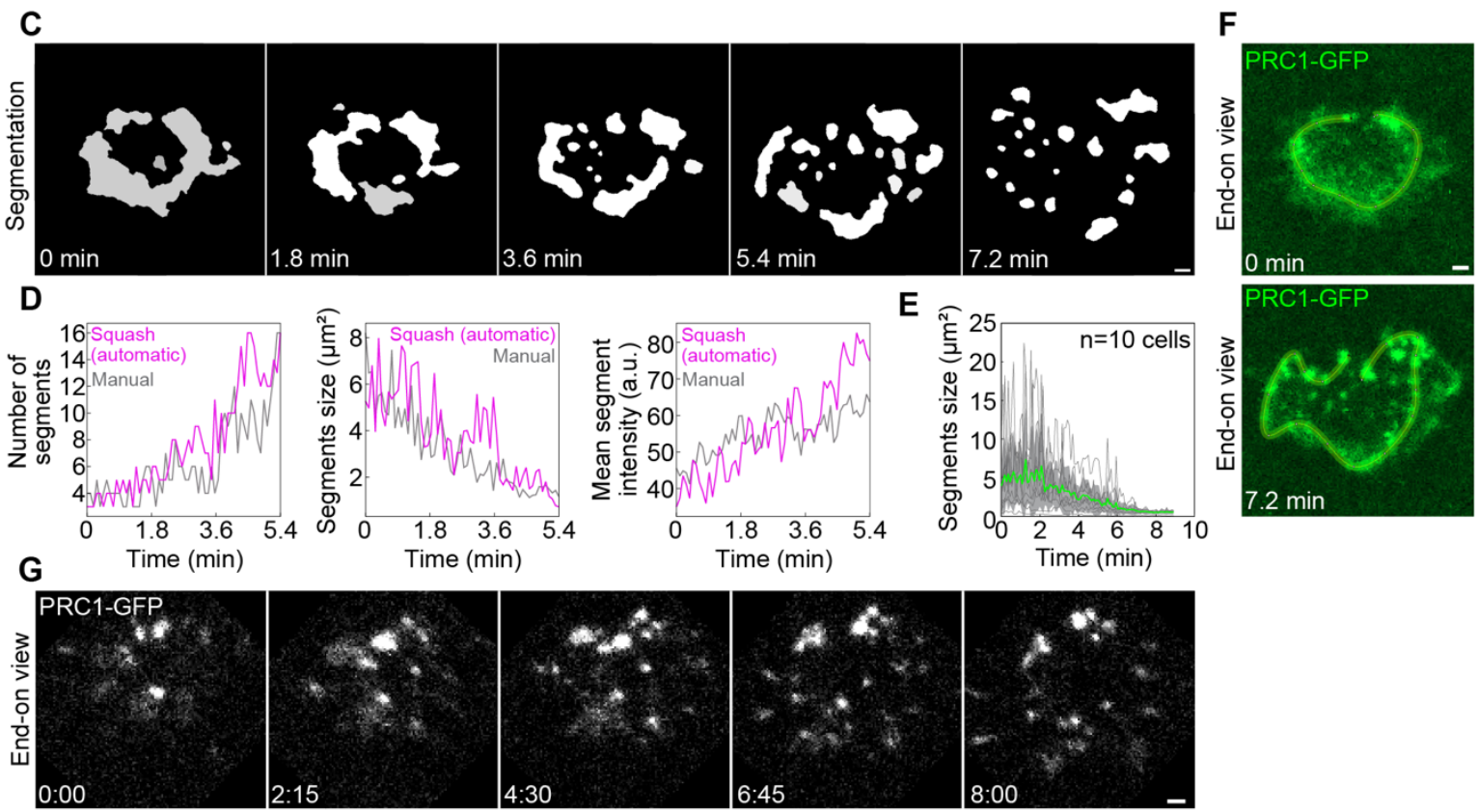

Time (min)
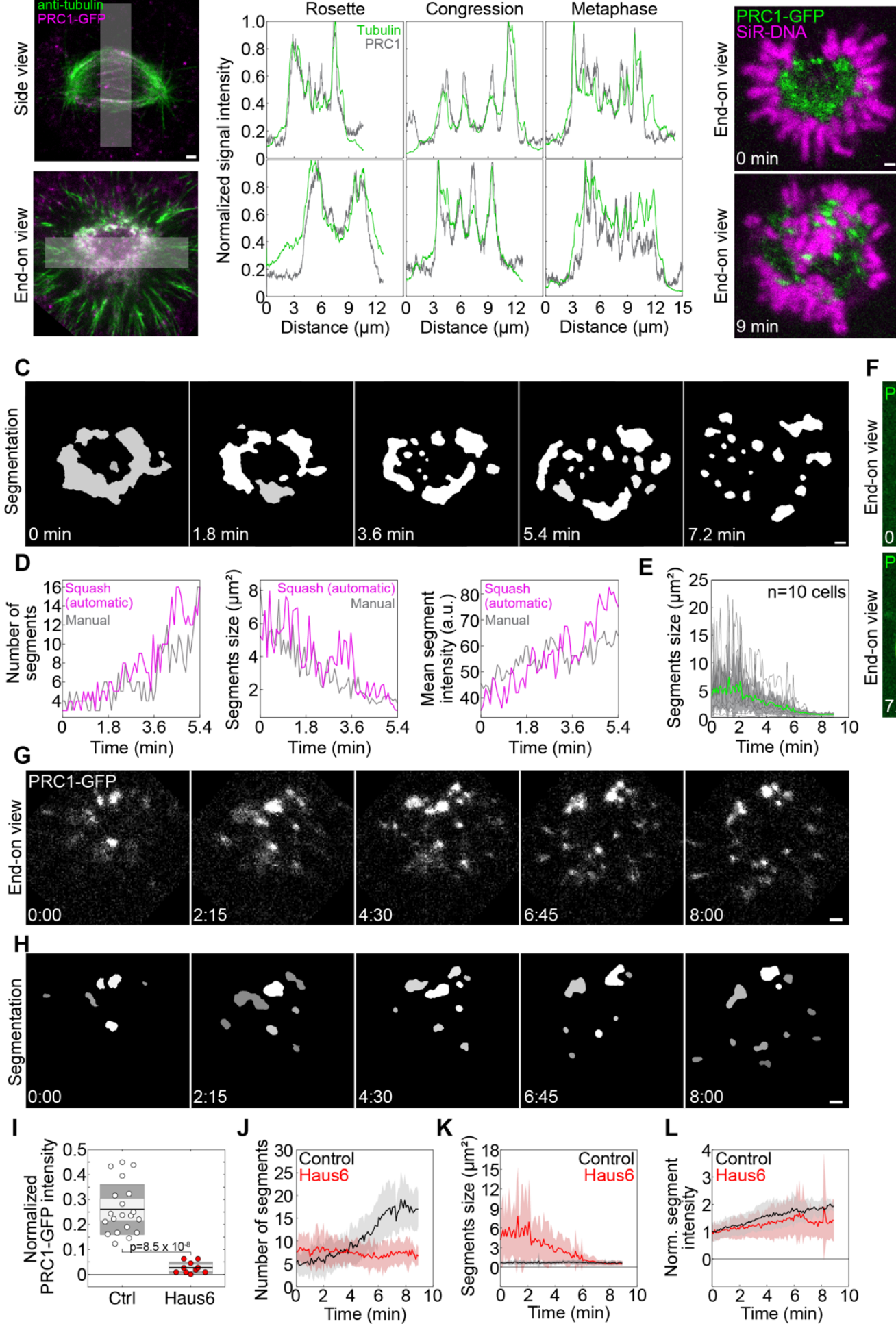

L

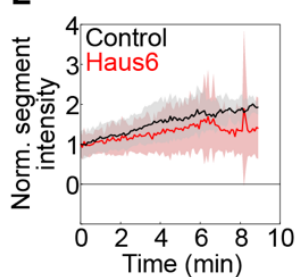

1184 
Extended Data Fig. 1: Additional characterization of the network-to-bundles transition of PRC1-crosslinked microtubules.

1187 a, STED images of tubulin (green) and confocal images of PRC1 (magenta) from Fig. 1a, with superimposed lines drawn in Fiji (left) used to measure line intensity profiles on horizontally oriented spindles (top), and vertically oriented spindles (bottom) in different phases as indicated (right). Both PRC1-GFP and anti-tubulin intensity profiles are shown. Intensity is normalized to the maximum value. Note the colocalization of the PRC1 and tubulin signal. $\mathbf{b}$, Midplane of a vertically oriented spindle in a HeLa-Kyoto BAC cell stably expressing PRC1-

1193 GFP (green) with DNA labeled by SiR-DNA (magenta), before (top) and after (bottom) 1194 antiparallel bundle formation (9 minutes). c, Squash segmentation results for time-lapse images 1195 of the midplane of a vertically oriented prometaphase spindle in a HeLa-Kyoto BAC cell stably 1196 expressing PRC1-GFP corresponding to Fig. 1b. d, Validation of the squash segmentation method by manual measurements on a representative spindle. Graphs show the number of segments, their size and mean intensity (from left to right). e, Mean size of PRC1 segments in the midplane over time in HeLa-Kyoto BAC cells stably expressing PRC1-GFP, obtained using squash segmentation. Gray lines represent individual cells, green line the mean and gray areas the standard deviation ( $\mathrm{n}=10$ cells in 10 independent experiments). $\mathbf{f}$, Lines drawn in Fiji along

1202 the periphery of the spindle midplane, where the PRC1 network is located in prometaphase, 1203 which were used to measure line intensity profiles in Fig. 1e,f. g, Time lapse images of the midplane (single plane) of a vertically oriented prometaphase spindle in a Haus6-depleted HeLa-Kyoto BAC cell stably expressing PRC1-GFP, starting with the prometaphase rosette.

1206 Time is shown in minutes:seconds. h, Squash segmentation results of the images shown in $\mathbf{g}$. 1207 i, Mean intensity of PRC1-GFP in a region on the opposite side of the bundles that lack 1208 chromosomes in the early prometaphase network in control $(n=20)$ and Haus6-depleted $(n=10)$ 1209 HeLa cells expressing PRC1-GFP. PRC1-GFP intensity is normalized to the cytoplasmatic 1210 PRC1-GFP intensity. j, Number of PRC1 segments in the midplane over time in Haus61211 depleted (red) and control (black, gray) HeLa-Kyoto BAC cells stably expressing PRC1-GFP 1212 ( $\mathrm{n}=10$ cells for control and 9 cells for Haus6 depletion), obtained using squash segmentation.

1213 Colored surfaces around the central lines (mean) represent standard deviation. $\mathbf{k}$, Segment size 1214 and $\mathbf{l}$, normalized segment intensity of PRC1-GFP segments from the same cells as in $\mathbf{j}$. All 1215 scale bars, $1 \mu \mathrm{m}$. 
A
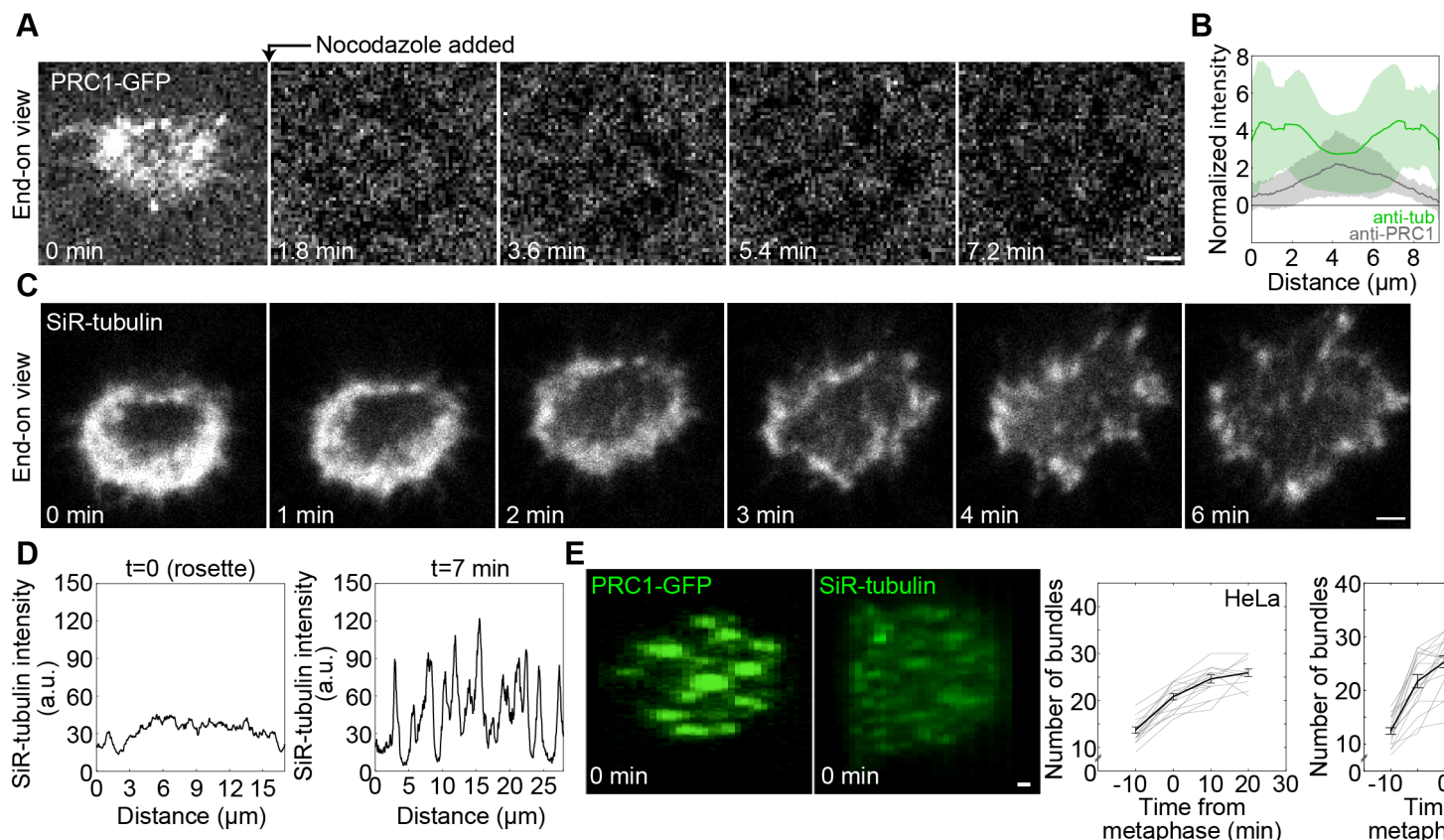

Extended Data Fig. 2: Additional characterization of PRC1 and tubulin during bundle

\section{formation.}

1220 a, Time lapse images of the midplane (single plane) of a vertically oriented prometaphase spindle in a HeLa-Kyoto BAC cell stably expressing PRC1-GFP, starting with the prometaphase rosette. Time when nocodazole was added is indicated. b, Intensity line plots of the anti-PRC1 (gray) and anti-tubulin (green) signal along the pole-to-pole axis in horizontally oriented early prometaphase spindles (rosette stage) of non-transfected immunostained HeLa cells, $\mathrm{n}=20$ cells from 2 independent immunostainings. Intensity was normalized to the cytoplasmatic anti-PRC1 and anti-tubulin intensity, respectively. Note that the PRC1 intensity is highest in the middle of the spindle, whereas tubulin intensity is highest away from the

1228 middle, i.e., close to the poles. c, Time lapse images of the midplane (single plane) of a 1229 vertically oriented prometaphase spindle in a HeLa-Kyoto BAC cell stably expressing PRC11230 GFP and stained with SiR-tubulin, starting with the prometaphase rosette. Only SiR-tubulin channel was imaged. d, SiR-tubulin intensity line plots along the periphery of the spindle midplane at $\mathrm{t}=0$ and $\mathrm{t}=7$ minutes from the cell shown in c. e, Reconstructed (rotated by 90 degrees) images of the midplane of a horizontally oriented spindle in a HeLa-Kyoto BAC cell

1234 stably expressing PRC1-GFP (left image) and in an hTERT-RPE1 cell expressing CENP-A1235 GFP and centrin1-GFP labeled with $\mathrm{SiR}$ tubulin (right image). Graphs show the number of 1236 bundles over time for the HeLa cell line (left, $n=12$ cells) and the RPE1 cell line (right, $n=16$ 
bioRxiv preprint doi: https://doi.org/10.1101/2022.02.25.481924; this version posted February 25, 2022. The copyright holder for this preprint (which was not certified by peer review) is the author/funder, who has granted bioRxiv a license to display the preprint in perpetuity. It is made available under aCC-BY-NC-ND 4.0 International license.

1237 cells), corresponding to the images on the left. Time zero is the beginning of metaphase. All 1238 scale bars, $1 \mu \mathrm{m}$. 
bioRxiv preprint doi: https://doi.org/10.1101/2022.02.25.481924 t this version posted February 25, 2022. The copyright holder for this preprint (which was not certified by peer review) is the author/funder, who has granted bioRxiv a license to display the preprint in perpetuity. It is made available under aCC-BY-NC-ND 4.0 International license.

Supplementary Figure 3

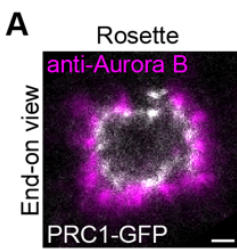

C
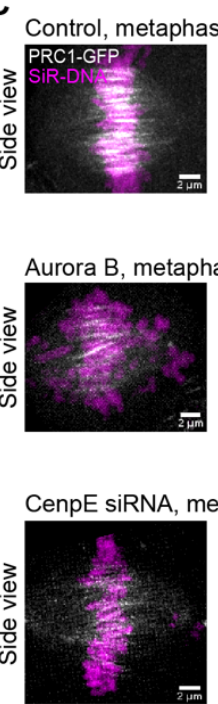

G

G Aurora B inhibitor barasertib added
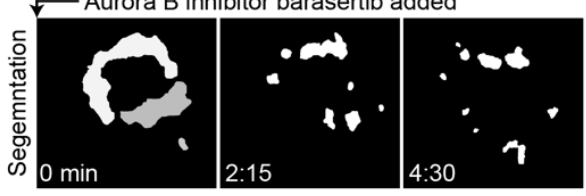

I
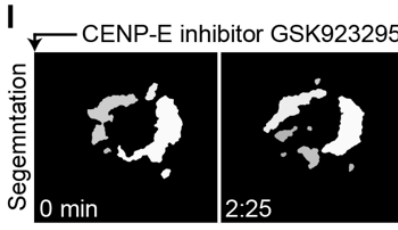

K Control

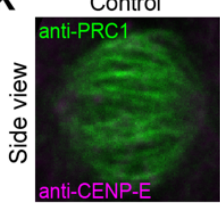

CENP-E
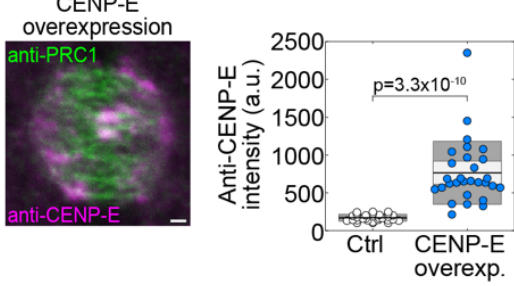

B

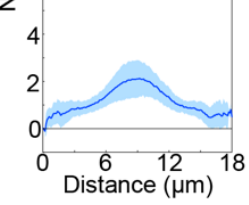

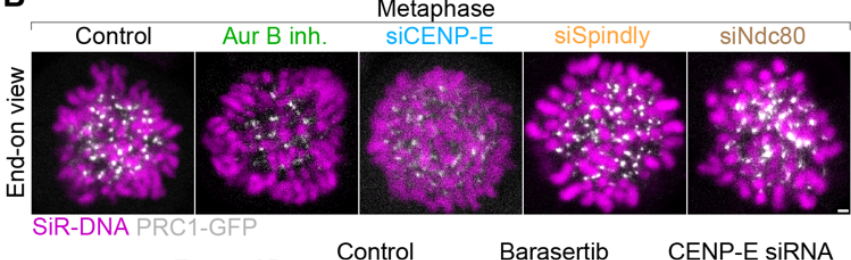

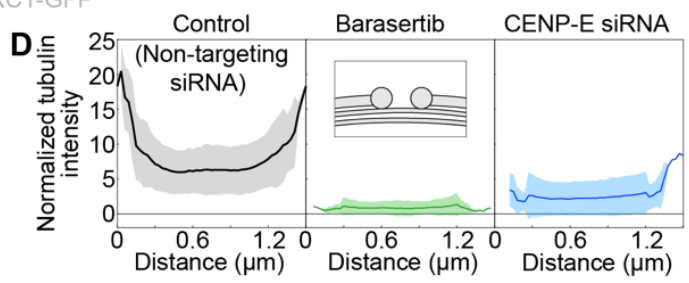

E
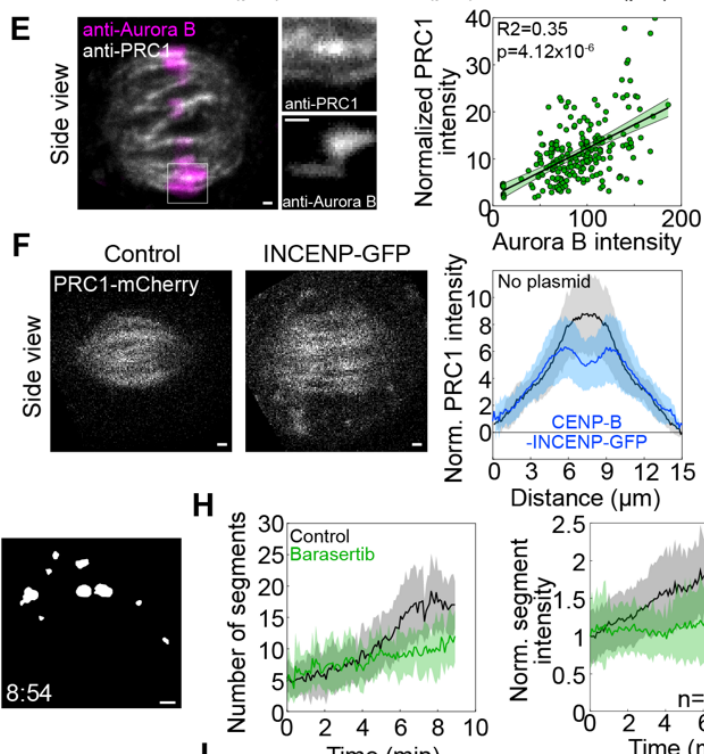
Distance $(\mu \mathrm{m})$

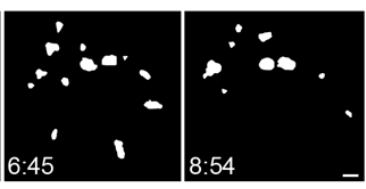

$\mathbf{J}$
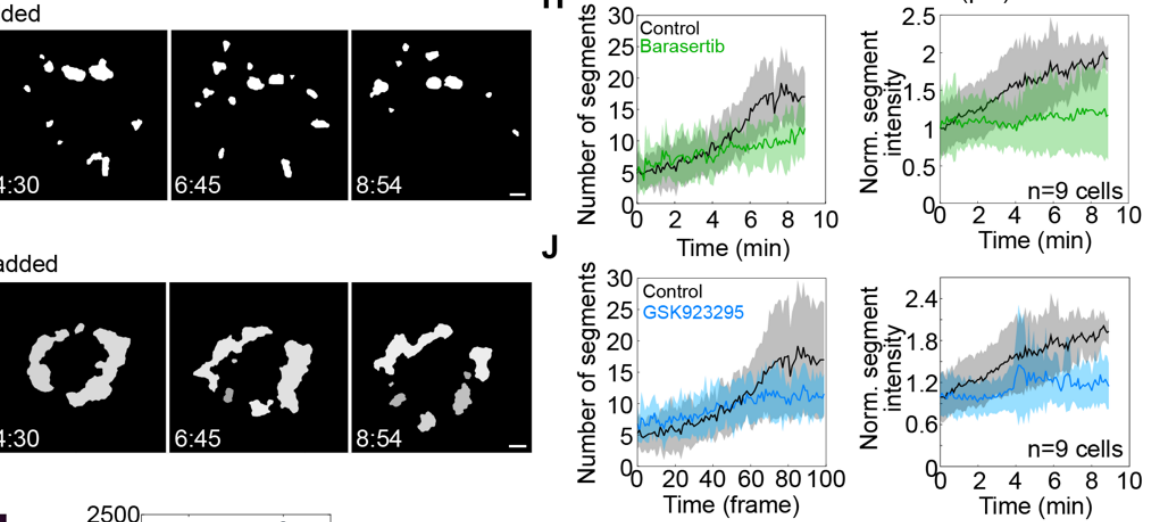

Extended Data Fig. 3: Additional characterization of the role of kinetochores in overlap bundle formation.

1243 a, Left, image of a vertically oriented prometaphase rosette immunostained for Aurora B

1244 (magenta) in a HeLa-Kyoto BAC cell stably expressing PRC1-GFP (white). Right, intensity

1245 line plot of PRC1-GFP (grey) and anti-Aurora B (green) along a line drawn across the spindle

1246 cross-section, passing through the center. Line thickness was $1 / 3$ of the spindle cross-section. 
Intensity is normalized to the maximum value for each channel. $\mathbf{b}$, Images of vertically oriented metaphase spindles in a HeLa-Kyoto BAC cell line stably expressing PRC1-GFP (white) from

1249 Fig. 2e. Chromosomes are labeled with SiR-DNA (magenta) to show the metaphase plate. c, 1250 Live-cell images of horizontally oriented metaphase spindles in HeLa-Kyoto BAC cells stably expressing PRC1-GFP (white) and stained with SiR-DNA (magenta). Control (top), Aurora B inhibition (middle) and CENP-E depletion (bottom). Maximal projections of 41 planes are shown. The corresponding intensity line profiles of PRC1-GFP are shown to the right, mean (central line) and standard deviation (colored surface). The line profiles were measured along a line drawn from pole to pole. Line thickness was $1 / 3$ of the spindle width. Intensity was normalized to the cytoplasmatic PRC1 intensity. Scale bars, $2 \mu \mathrm{m}$. Number of cells: 56 for control, 32 for Aurora B inhibition, 43 for CENP-E siRNA. All measurements are from 3 independent experiments. d, Intensity line profiles corresponding to anti-tubulin signal in the bridging fiber from Fig. 2f,g and Fig. 3b,c; mean (central line) and standard deviation (colored surface); $\mathrm{n}=22$ bundles from 14 cells for control, 11 bundles from 9 cells for Aurora B inhibition, and $\mathrm{n}=10$ bundles from 7 cells for CENP-E siRNA, from 3 independent immunostainings. Intensity was normalized to the cytoplasmatic anti-tubulin intensity. e, Image of a spindle immunostained for Aurora B (magenta) and PRC1 (white) in an untransfected HeLa cell in metaphase. Sum of 5 planes is shown. Enlargements of anti-PRC1 and anti-Aurora B (both in white) within the square marked on the main image are shown to the right. Graph shows correlation between anti-PRC1 and anti-Aurora B signal on the corresponding centromere, $\mathrm{n}=404 \mathrm{PRC} 1$-Aurora $\mathrm{B}$ pairs from 26 cells. Intensity was normalized to the cytoplasmatic anti-PRC1 and anti-Aurora B intensity, respectively. $\mathbf{f}$, Live cell images of metaphase spindles in HeLa cells transfected with PRC1-mCherry (white) in a control cell (left image) and a cell transfected with CENP-B-INCENP-GFP construct (right image). Maximal projections of 41 planes are shown. Graph shows intensity line plots of PRC1-mCherry along the spindle main axis, mean (central line) and standard deviation (colored surfaces), $n=29$ cells for control and $n=28$ for CENP-B-INCENP-GFP, 3 independent experiments per condition. Intensity was normalized to the cytoplasmatic PRC1 intensity. g,

1275 Squash segmentation results for time-lapse images of the midplane of a Barasertib-treated 1276 vertically oriented prometaphase spindle in a HeLa-Kyoto BAC cell stably expressing PRC11277 GFP, corresponding to Fig. 2j. h, Number of PRC1 segments (left) and normalized mean 1278 intensity of PRC1 segments (normalized to the intensity at $\mathrm{t}=0$, right) over time from squash 1279 segmentation in control and Barasertib-treated (i.e., Aurora B-inhibited) cells, as indicated. 1280 Colored surfaces around the central lines (mean) represent standard deviation, $\mathrm{n}=9$ cells from 
12819 independent experiments. $\mathbf{i}$, Squash segmentation results and $\mathbf{j}$, the related quantification for 1282 cells treated with the CENP-E inhibitor GSK923295, corresponding to Fig. 3f. Legend as in $\mathbf{g}$ 1283 and $\mathbf{h}$, respectively, $\mathrm{n}=9$ cells from 9 independent experiments. $\mathbf{k}$, Images of horizontally 1284 oriented spindles in metaphase, immunostained for PRC1 (green) and CENP-E (magenta), in 1285 a control HeLa cell (left image) and after CENP-E overexpression (right image). Graph shows 1286 total CENP-E intensity on the spindle in control cells and after CENP-E overexpression, $\mathrm{n}=29$ 1287 cells in each condition from 3 independent experiments. The black line shows the mean; the 1288 light and dark grey areas mark 95\% confidence interval on the mean and standard deviation, 1289 respectively. Note that the overexpression level is roughly 5-fold. In all controls for siRNA 1290 experiments the cells were transfected with nontargeting siRNA. All scale bars except in c, 1 $1291 \mu \mathrm{m}$. 


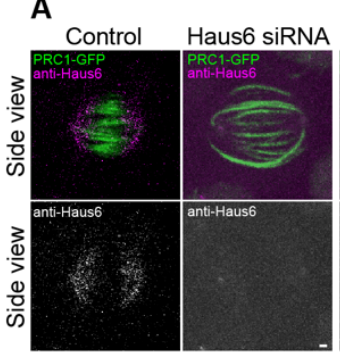

B
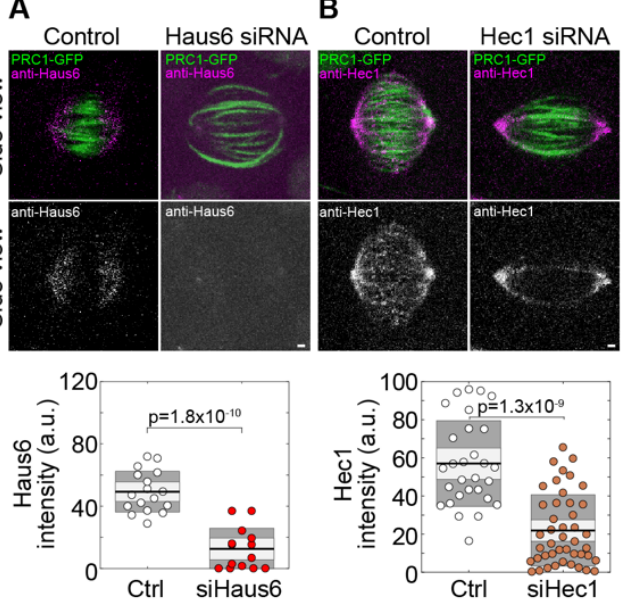

Supplementary Figure 4
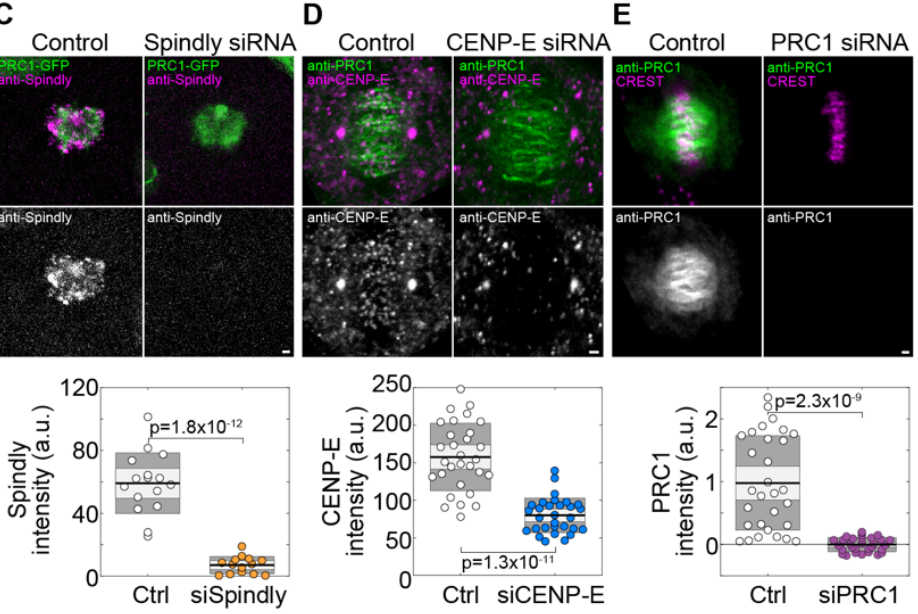

1294 Extended Data Fig. 4: Quantification of protein depletion by immunostaining.

1295 In each panel the images show control and depleted spindles, as indicated, with merged

1296 channels in the first row and only the channel of the depleted protein (white) in the second row.

1297 The graph under the images shows the quantification of the total signal intensity of the 1298 indicated protein on the spindle in control and depleted cells. For Hecl depletion and the 1299 corresponding control, the total intensity of anti-Hecl signal was measured in a region 1300 encompassing all DNA, i.e., excluding the poles. In a-c, HeLa-Kyoto BAC cell line stably 1301 expressing PRC1-GFP was used, whereas in d,e, untransfected HeLa cells were used. The 1302 number of cells was: (a) control, $n=17$, and Haus6 depletion, $n=14$, (b) control, $n=29$, and Hec 1 1303 depletion, $\mathrm{n}=44$, (c) control, $\mathrm{n}=16$, and Spindly depletion, $\mathrm{n}=14$, (d) and (e), $\mathrm{n}=30$ for each 1304 condition. Mean silencing was: (a) 74\%, (b) 62\%, (c) $90 \%$, (d) $51 \%$, (e) $\sim 100 \%$ when the 1305 background signal is subtracted (data shown in panel e) and 86\% without background 1306 subtraction (data not shown). In all control experiments the cells were transfected with nontargeting siRNA. All scale bars, $1 \mu \mathrm{m}$. 

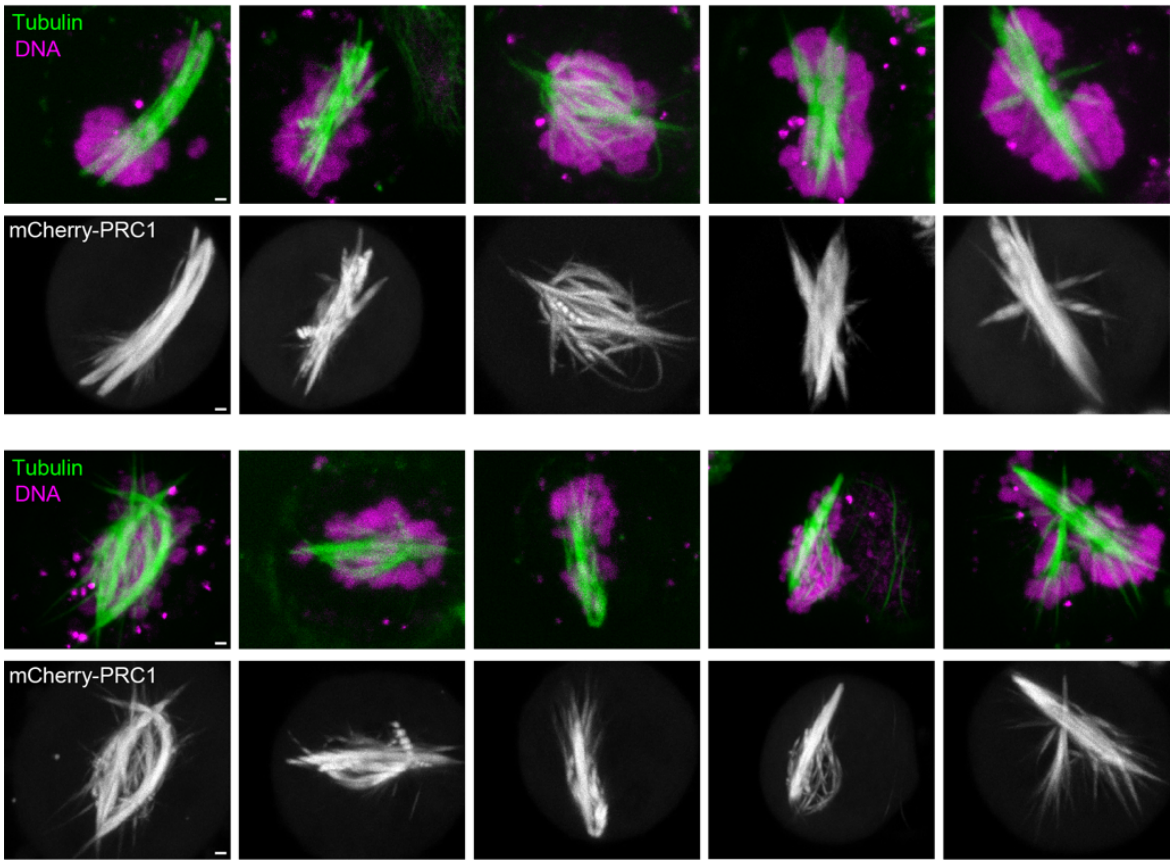

1310 Extended Data Fig. 5: Additional images of spindles in cells with overexpressed PRC1.

1311 Examples of 10 spindles with overexpressed PRC1 in HeLa-TDS cells. Merged images show

1312 microtubules (SiR-tubulin, green) and DNA (Hoechst 33342, magenta), and the corresponding

1313 single-channel images below the merged ones show PRC1-mCherry (white). Scale bars, $1 \mu \mathrm{m}$. 


\section{Supplementary Video 1. Overlap bundle formation during early prometaphase.}

1317 Time lapse of a midplane of a vertically oriented prometaphase spindle in a HeLa-Kyoto BAC 1318 cell stably expressing PRC1-GFP starting with prometaphase rosette. A single plane is shown.

1319 Time interval between acquired images is 5.4 seconds. Time is shown in minutes:seconds.

1320 Scale bar, $1 \mu \mathrm{m}$.

\section{Supplementary Video 2. Bundle formation after Haus6 (augmin subunit) depletion.}

1323 Time lapse of a midplane of a vertically oriented prometaphase spindle in a Haus6-depleted 1324 HeLa-Kyoto BAC cell stably expressing PRC1-GFP starting with prometaphase rosette. A single plane is shown. Time interval between acquired images is 5.4 seconds. Time is shown in minutes:seconds. Scale bar, $1 \mu \mathrm{m}$.

\section{Supplementary Video 3. Bundle formation after Aurora B inhibition.}

1329 Time lapse of a midplane of a vertically oriented prometaphase spindle in a HeLa-Kyoto BAC 1330 cell stably expressing PRC1-GFP starting with prometaphase rosette. Barasertib was added just before the beginning of the movie. A single plane is shown. Time interval between acquired images is 5.4 seconds. Time is shown in minutes:seconds. Scale bar, $1 \mu \mathrm{m}$.

\section{Supplementary Video 4. Bundle formation after CENP-E inhibition.}

1335 Time lapse of a midplane of a vertically oriented prometaphase spindle in a HeLa-Kyoto BAC cell stably expressing PRC1-GFP starting with prometaphase rosette. GSK923295 was added just before the beginning of the movie. A single plane is shown. Time interval between acquired images is 5.4 seconds. Time is shown in minutes:seconds. Scale bar, $1 \mu \mathrm{m}$.

\section{Supplementary Video 5. Movement of overlap bundles during prometaphase.}

1341 Left, time lapse of a midplane of a vertically oriented prometaphase spindle in a HeLa-Kyoto

1342 BAC cell stably expressing PRC1-GFP starting with prometaphase rosette. The images were translated so that the position of the two bright bundles that are initially found in the upper region of the spindle is roughly constant. A single plane is shown. Time interval between acquired images is 2.7 seconds. Right, trajectories of the bundles from the movie on the left. Time is shown in minutes:seconds. Scale bar, $1 \mu \mathrm{m}$. 
1348 Supplementary Video 6. Chromosome congression to the spindle midplane causes spindle 1349 widening, end-on view.

1350 Time lapse of the central part of a vertically oriented prometaphase spindle in a HeLa-Kyoto

1351 BAC cell stably expressing PRC1-GFP (green) starting with prometaphase rosette. DNA is 1352 stained with SiR-DNA (magenta). Maximum projection of 3 middle planes is shown. Time 1353 interval between acquired images is 30 seconds. Time is shown in minutes:seconds. Scale bar, $13541 \mu \mathrm{m}$.

1355

1356 Supplementary Video 7. Chromosome congression to the spindle midplane causes spindle 1357 widening, side view.

1358 Time lapse of a horizontally oriented prometaphase spindle in a HeLa-Kyoto BAC cell stably 1359 expressing PRC1-GFP (green) starting with prometaphase rosette. DNA is stained with SiR1360 DNA (magenta). Sum of 41 planes is shown. Time interval between acquired images is 30 1361 seconds. Time is shown in minutes:seconds. Scale bar, $1 \mu \mathrm{m}$. 